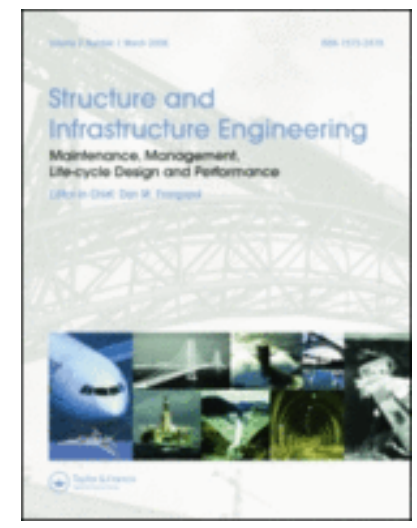

\title{
Finite-element-model updating of civil engineering structures using a hybrid UKF-HS algorithm
}

\begin{tabular}{|r|l|}
\hline Journal: & Structure and Infrastructure Engineering \\
\hline Manuscript ID & NSIE-2019-0384.R1 \\
\hline Manuscript Type: & Original Paper \\
\hline Author: & 01-Dec-2019 \\
\hline Complete List of Authors: & $\begin{array}{l}\text { Naranjo-Perez, Javier; Universidad de Sevilla Escuela Superior de } \\
\text { Ingenieros, Department of Continuum Mechanics and Structural Analysis } \\
\text { Jimenez-Alonso, Javier Fernando; Polytechnic University of Madrid } \\
\text { Superior Technical School of Civil Engineering, Department of Continum } \\
\text { Mechanics and Structures } \\
\text { Pavic, Aleksandar; University of Exeter College of Engineering } \\
\text { Mathematics and Physical Sciences, ; } \\
\text { Saez, Andres; Universidad de Sevilla Escuela Superior de Ingenieros, } \\
\text { Department of Continuum Mechanics and Structural Analysis }\end{array}$ \\
\hline Keywords: & $\begin{array}{l}\text { Civil \& Structural Engineering: Structural Engineering, Finite element } \\
\text { method, Dynamic analysis, Non-destructive tests, Optimization }\end{array}$ \\
\hline &
\end{tabular}

\section{SCHOLARONE \\ Manuscripts}




\section{Finite-element- model updating of civil engineering structures using a hybrid UKF-HS algorithm}

Finite-element-model updating allows reducing the discrepancies between the numerical and the experimental dynamic behaviour of civil engineering structures. Among the different methods to tackle the updating problem, the maximum likelihood method has been widely used for practical engineering applications. In this method, the updating problem is transformed into an optimization problem where the relative differences between the numerical and experimental modal properties of the structure are reduced via the modification of the most relevant physical parameters of the model. However, this method often presents the drawback of requiring high simulation times in order to perform the updating process when dealing with complex structures. To overcome this limitation, in this paper a novel hybrid Unscented Kalman Filter Harmony Search (UKF-HS) algorithm is proposed and its implementation details are discussed. In order to validate such hybrid algorithm and further illustrate its performance, the finite-element-model updating of a benchmark footbridge is performed using two different approaches (single-objective and multi-objective) and three different computational algorithms, namely: (i) genetic algorithms; (ii) harmony search; and (iii) the novel UKF-HS hybrid algorithm. The obtained results reveal that the proposed hybrid algorithm may be considered as an adequate alternative tool to efficiently perform the finite-element-model updating of civil engineering structures in practical engineering applications.

Keywords: finite-element-model updating; unscented Kalman filter, harmony search; genetic algorithm; hybrid algorithms; maximum likelihood method.

\section{Introduction}

Finite-element-model updating allows reducing the discrepancies between the predictions provided by numerical models and the real behaviour of civil engineering structures (Friswell and Mottershead, 1995). In this manner, the resulting finiteelement-models will closely reproduce the actual behaviour of the structure. For this reason, finite-element-model updating has been widely used in engineering practice to conduct structural assessment analyses (Fritzen et al., 1998; Fan and Qiao, 2011), in damage detection applications (Teughels et al., 2002; Wang et al., 2014) or for structural health monitoring of existing structures (Beck, et al., 2001).

Finite-element-model updating basically focuses on obtaining the values of the most relevant physical parameters of the structure which minimize the difference between the numerical and experimental behaviour of the structure (Mottershead et al., 
2011). For this purpose, the modal properties of the structure are usually considered to characterize its behaviour. In this manner, the finite-element-model updating problem can be transformed into a parameter identification problem (Friswell and Mottershead, 1995). In order to address this identification problem, estimators are normally considered (Marwala, 2010). Among the different estimators, two have been widely used in practical engineering applications: (i) Bayesian inference; and (i) maximum likelihood method.

Bayesian inference (Marwala et al., 2016) allows finding the posterior probability density function of the considered parameters via a statistical method based on Bayes' theorem. According to this theorem, the a priori information on the probability density function of the parameters can be updated based on information provided by the observations (experimental data). The expectation of the posterior probability density function may be evaluated through multi-dimensional integrals. As these integrals do not usually have analytical solutions, numerical approximations (Beck and Katafygiotis, 1998) and stochastic simulation methods (Papadimitriou et al., 2018) have been widely employed to this end. Between these two methods, stochastic simulations have been extensively used due to its ease of implementation and higher accuracy (Cheung and Beck, 2009). Stochastic simulations are based on the generation of samples (sampling techniques). Among these sampling methods, the so-called Markov Chain Monte Carlo (MCMC) methods have been normally used to solve the finite-element-model updating problem (Beck and Au, 2002). Different algorithms have been developed to perform a MCMC simulation. Among these algorithms, some outstanding examples for Bayesian finite-element-model updating applications are (according to the best of the authors' knowledge): (i) the Metropolis-Hastings algorithm (Robert and Casella, 1999); the Gibbs sampling algorithm (Ching et al., 2006); the transitional MCMC algorithm (Ching and Chen, 2007); and the Hamiltonian Markov Chain algorithm (Cheung and Beck, 2009). All these Bayesian methods present as main advantage that they provide the overall probabilistic distribution of the considered physical parameters rather than a point estimation of their expected value. Nevertheless, they present the drawback of the high simulation time required to perform the finiteelement-model updating when tackling complex civil engineering structures (JiménezAlonso et al., 2019). In particular for the case when the number of unknown physical parameters to be updated is large (Astroza et al., 2019; Bartilson et al., 2019; Vakilzadeh et al., 2014), or when it is not possible to implement a parallelizable Bayesian computational approach (Astroza et al., 2019; Cheung and Beck, 2009; Papadimitriou et al., 2018;).

On the other hand, the maximum likelihood method focuses on estimating the expected value of the considered physical parameters via the maximization of the likelihood between the numerical and experimental modal properties of the structure. Under the assumption of a normal distribution of the errors, this method is equivalent to the ordinary least squares estimator. Thus, the maximum likelihood method allows formulating the finite-element-model updating problem as an optimization problem (Wang et al., 2011). Hence, the objective function is defined in terms of the sum of the squares of the relative differences between the numerical and experimental modal properties of the structure. A limitation of this method is that, in contrast to Bayesian inference, only the expected values of the physical parameters can be obtained. Although this fact makes Bayesian inference a more robust estimator for updating problems, the high simulation time required to perform the updating process of complex civil engineering structure has motivated a more intensive use of the maximum likelihood method for practical engineering applications. Although computational times 
involved in Bayesian updating may significantly be reduced by parallelization, the ease of implementation of the maximum likelihood method for such practical applications has prompted us to tackle the updating problem using these later algorithms.

In order to solve the resulting optimization problem, either local or global optimization algorithms can be employed (Nocedal and Wright, 1999). As the relationship between the considered physical parameters and the modal properties of the structure is clearly nonlinear, multiple optimums are normally expected in the objective function of this problem. Provided that local optimization algorithms may have difficulties to find the global optimum under such scenario, global optimization algorithms are usually employed to solve the updating problem. To this end, computational intelligence algorithms, like genetic algorithms (GA) and artificial neural networks (ANN) have been widely used for practical engineering applications (Marwala, 2010). Both algorithms have shown their efficiency when solving nonlinear optimization problems (Koh and Perry, 2009; Levin and Lieven, 1998a). For instance, GA have been successfully employed to assess the structural behaviour of existing bridge structures (Jiménez-Alonso and Sáez, 2016) or to detect damage in civil engineering structures (Hao and Xia, 2002). ANN have as well been implemented to conduct the finite-element-model updating of a wide variety of structural systems (Lu and Tu, 2004; Hasançebi and Dumlupınar, 2013; Guo et al., 2017). Many other success stories are scattered in the scientific literature for finite element updating.

The main limitation of these computational algorithms is the high simulation time required to perform the finite-element-model updating when the complexity of the structure increases. Two trends have been considered to overcome this drawback (if we keep parallelization aside in this review): (i) the implementation and validation of more recent global optimization algorithms and (ii) the hybridization between local and global optimization algorithms.

As representative examples of the first trend, Levin and Lieven (1998b) performed a comparison between the performance of the GA and the simulated annealing (SA) to solve the finite-element-model updating problem of a flat plate wing; Perera et al. (2010) compared the results obtained between GA and the particle swarm optimization (PSO) for the finite-element-model updating of a one-story reinforced concrete frame.

On the other hand, two outstanding examples of the second trend can be remarked: (i) the work reported by Shabbir and Omenzetter (2015), who proposed a hybrid sequential niche-PSO algorithm to perform the finite-element-model updating of a footbridge, and (ii) the study provided by Astroza et al. (2016), who implemented a hybrid unscented Kalman filter-SA algorithm to perform the finite-element-model updating of a steel frame structure.

In this paper, a novel computational algorithm devised to improve the efficiency of finite-element-model updating of civil engineering structures is proposed, implemented and further validated. The design of this new algorithm employs the maximum likelihood approach and stems from the two trends mentioned above.

On the one hand, as basis of this new algorithm, the Harmony Search (HS) global optimization algorithm is adopted. The harmony search algorithm is based on the improvisation process in which musicians seek harmony (Geem et al., 2001). This algorithm has been applied efficiently in multiple practical engineering applications (Yang and Koziel, 2011). However, according to the best of the authors' knowledge, it 
has been rarely applied for the finite-element-model updating of civil engineering structures (Fadel et al., 2012; Kaveh et al., 2014).

On the other hand, the performance of the original HS algorithm is improved via its hybridization with a local optimization algorithm: the so-called Unscented Kalman Filter (UKF) is considered herein. This algorithm belongs to the Kalman filter family (Kalman, 1960) which originally focused on estimating the means and the covariance of the constitutive parameters of a linear system through a recursive process with two steps: (i) the prediction; and (ii) the correction steps. Subsequently, the original Kalman filter was adapted to nonlinear systems (Jazwinski, 1970) through the so-called Extended Kalman Filter (EKF). The basic idea of this algorithm is to linearize locally the nonlinear function, which represents the behaviour of the system, before applying the Kalman filter. Thus, one of the main drawbacks of this algorithm is that it only allows computing a first order approximation of the nonlinear function. In order to overcome this limitation, the Unscented Kalman Filter (UKF) was subsequently proposed by Julier and Uhlmann (1997). This algorithm defines a set of sampling points which allow estimating the mean and covariance of the constitutive parameters after their propagation through the nonlinear system. The direct estimation of these statistical properties allows computing a second order approximation (or even a third order approximation in case of a Gaussian random vector is propagated) of the nonlinear function (Van Der Merwe, 2004; Nguyen and Nestorović, 2015).

The resulting hybrid UKF-HS algorithm is then formulated to tackle two types of optimization problems: (i) single-objective and (ii) multi-objective approaches. The implementation details are next thoroughly presented and discussed. The performance of the new algorithm is validated and further compared with the results of other two global computational intelligence algorithms (GA and HS). To this end, the finite element model updating of a benchmark reconfigurable steel footbridge (Hudson and Reynolds, 2017) located at the laboratory of the Vibration Engineering Section of the University of Exeter (U.K.) is considered. The experimental modal properties of the structure were obtained via the signal processing of the records obtained during a forced vibration test (Maia and Silva, 1997).

The paper is organized as follows. First, some basics about finite-element-model updating under the maximum likelihood method are presented. Subsequently, the three considered computational intelligence algorithms (GA, HS, UKF-HS) are described in detail, with special emphasis on the newly proposed UKF-HS algorithm. Subsequently, the performance of the three mentioned algorithms is compared when they are implemented for the finite-element-model updating of a laboratory footbridge. Finally, some concluding remarks are drawn to close the paper in the fifth section.

\section{Basics of Finite-element-model Updating under the Maximum Likelihood}

\section{Method}

As indicated above, the finite-element-model updating problem may be considered as a parameter identification problem in which the values of the most relevant physical parameters of the structure are to be estimated through the minimization of the differences between the numerical and experimental modal properties of the structure (Mottershead et al., 2011). Different estimators are normally used to solve this parameter identification problem. Among them, the maximum likelihood method has been widely used to perform the finite-element-model updating of civil engineering structures (Marwala, 2010) and it will be the approach considered 
herein.

In this manner, the finite-element-model updating problem is transformed into an optimization problem, where the updated model follows from iterative modifications of the most relevant physical parameters, until an optimal solution is obtained. The objective function of such optimization problem may be defined in terms of the square relative differences (residuals) between the numerical and the experimental modal properties of the structure (natural frequencies and vibration modes). As design parameters of this problem, the most relevant physical parameters of the model are considered. Provided that the relationship between the residuals and the design parameters is nonlinear, global optimization algorithms are needed to perform the optimization process (Nocedal and Wright, 1999). Additionally, a search domain is usually established to guarantee that the solution obtained retains its physical meaning. Two approaches can be considered to define the objective function: (i) the singleobjective approach; and (ii) the multi-objective approach.

Under the single-objective approach, the optimization problem consists in minimizing a single-objective function defined in terms of the weighted residuals between the numerical and experimental modal properties of the structure. These weights take into account the relative contribution of each residual. The value of these weights can be established either by a statistical criterion (Friswell and Mottershead, 1995) or by a correlation criterion (Teughels et al., 2002). According to the first criterion, the weights are determined in terms of the uncertainty associated with the estimates of the experimental modal properties of the structure. According to the second criterion, the weights are defined by an iterative process (trial and error) which ensures the best agreement between the numerical and experimental modal properties of the structure. The second criterion will be considered herein.

Under the multi-objective approach, the optimization problem consists in minimizing a multi-objective function defined by several functional components. The overall objective function is normally defined by two functional components for practical engineering applications (Jin et al., 2014). One component may be defined in terms of the residuals associated with the natural frequencies, $r_{f, j}(\boldsymbol{\theta})$, whilst the other component may be defined in terms of the residuals associated with the vibration modes, $r_{m, j}(\boldsymbol{\theta})$ ( $j$ being the considered vibration mode). In this case, no weights are needed to define the objective function. However, a set of possible solutions (the socalled Pareto front) is obtained as result of the optimization process. Hence, a subsequent decision making problem must be addressed in order to select the best solution among the different elements of the Pareto front. Among the different criteria provided in literature (Infantes et al., 2019), the normal boundary intersection (NBI) method has been considered herein (Deb and Gupta, 2011).

Residuals $r_{f, j}(\boldsymbol{\theta})$ and $r_{m, j}(\boldsymbol{\theta})$ can be defined as follows:

$$
\begin{gathered}
r_{f, j}(\boldsymbol{\theta})=\frac{f_{\text {num }, j}(\boldsymbol{\theta})-f_{\text {exp }, j}}{f_{\text {exp }, j}} \quad j=1,2,3, \ldots n_{f} \\
r_{m, j}(\boldsymbol{\theta})^{2}=\frac{\left(1-\sqrt{M A C_{j}(\boldsymbol{\theta})}\right)^{2}}{M A C_{j}(\boldsymbol{\theta})} \quad j=1,2,3, \ldots n_{f}
\end{gathered}
$$

where $\boldsymbol{\theta}$ is a vector containing the most relevant physical parameters of the model; $n_{f}$ is the total number of vibration modes considered to conduct the updating; $f_{n u m, j}$ and 
$f_{\text {exp, } j}[\mathrm{~Hz}]$ are the numerical and experimental natural frequencies $(j$ being the number of the considered vibration mode), respectively; and $M A C_{j}$ denotes the Modal Assurance Criterion (Allemang and Brown, 1982) which assesses the correlation between the numerical and experimental vibration modes. The $M A C_{j}$ ratio can be expressed as follows:

$$
M A C_{j}(\boldsymbol{\theta})=\frac{\left(\phi_{\mathrm{num}, j}(\boldsymbol{\theta}) \cdot \phi_{\mathrm{exp}, j}\right)^{2}}{\left(\phi_{\mathrm{num}, j}^{T}(\boldsymbol{\theta}) \cdot \phi_{\mathrm{num}, j}(\boldsymbol{\theta})\right) \cdot\left(\phi_{\mathrm{exp}, j}^{T} \cdot \phi_{\mathrm{exp}, j}\right)}
$$

with $\phi_{n u m, j}$ and $\phi_{\text {exp,j }}$ being the numerical and experimental vibration modes, respectively.

On the one hand, the formulation of the finite-element-model updating problem using the single-objective approach can be formulated as follows:

$$
\begin{gathered}
\min f(\boldsymbol{\theta})=\frac{1}{2}\left[\sum_{j}^{n_{f}} w_{f, j} \cdot r_{f, j}(\boldsymbol{\theta})^{2}\right]^{1 / 2}+\frac{1}{2}\left[\sum_{j}^{n_{f}} w_{m, j} \cdot r_{m, j}(\boldsymbol{\theta})^{2}\right]^{1 / 2} \\
\text { subject to }\left\{\boldsymbol{\theta}_{\mathbf{l}} \leq \boldsymbol{\theta} \leq \boldsymbol{\theta}_{\mathbf{u}} ; \sum_{j}^{n_{f}} w_{f, j}+w_{m, j}=1\right\}
\end{gathered}
$$

$w_{f}$ and $w_{m}$ being the weights associated with the residuals of the natural frequencies and vibrations modes; and $\boldsymbol{\theta}_{\mathbf{l}}$ and $\boldsymbol{\theta}_{\mathbf{u}}$ being the lower and upper bounds of the search domain of the considered physical parameters, respectively.

On the other hand, the formulation of the finite-element-model updating problem using the multi-objective approach can be formulated as follows:

$$
\min f(\boldsymbol{\theta})=\min \left(f_{1}(\boldsymbol{\theta}) \quad f_{2}(\boldsymbol{\theta})\right) \text { where }\left\{\begin{array}{l}
f_{1}(\boldsymbol{\theta})=\frac{1}{2}\left[\sum_{j}^{n_{f}} r_{f, j}(\boldsymbol{\theta})^{2}\right]^{1 / 2} \\
f_{2}(\boldsymbol{\theta})=\frac{1}{2}\left[\sum_{j}^{n_{f}} r_{m, j}(\boldsymbol{\theta})^{2}\right]^{1 / 2}
\end{array}\right.
$$

$$
\text { subject to: } \boldsymbol{\theta}_{\mathbf{l}} \leq \boldsymbol{\theta} \leq \boldsymbol{\theta}_{\mathbf{u}}
$$

In summary, a typical finite-element-model updating consists of the following steps (Marwala, 2010): (i) the experimental modal properties of the structure are identified via the signal processing (either experimental or operational modal analysis may be employed to this end) of the records obtained during a vibration test (either forced vibration test or ambient vibration test); (ii) the numerical modal properties of the structure are obtained via a modal analysis based on a finite-element model; (iii) the most relevant physical parameters of the model are modified iteratively (using a global optimization algorithm); (iv) the objective function is evaluated; and (v) this iterative process is repeated until some convergence criterion is met. As result of this process, either a vector of updated parameters (single-objective approach) or a Pareto front (multi-objective approach) is obtained. In the latter case, a subsequent decision making problem must be addressed in order to select the vector of updated parameters. 


\section{Computational Intelligence Algorithms for the Finite-element model}

\section{Updating of Civil Engineering Structures.}

The finite-element-model updating problem using the maximum likelihood method is usually tackled in practical engineering applications by employing computational intelligence algorithms (Marwala, 2010). These algorithms present as main advantage that the solution obtained is a global optimum of the objective function. However, they have the drawback of the high simulation time required to perform the updating process, especially when the complexity of the model increases. In order to overcome this limitation, several strategies have been adopted, as summarized in the introduction: from parallelizing the algorithms to the development of alternative global optimizers or the devising of hybrid local-global algorithms. In this paper, a novel hybrid Unscented Kalman Filter-Harmony Search (UKF-HS) algorithm is proposed and validated. This hybrid algorithm takes advantage of both the acceleration scheme provided by the unscented Kalman filter algorithm and the global search characteristics of the harmony search algorithm.

The performance of this hybrid algorithm is assessed by comparing the results of the finite-element-model updating of a laboratory footbridge conducted using three different computational intelligence algorithms: (i) the classic genetic algorithms (GA); (ii) the harmony search algorithm (HS); and (iii) the novel hybrid UKF-HS algorithm. Further comparison of the performance of the new algorithm with the quite numerous optimizers available in the literature is out of the scope of this paper. However, it is relevant to point out here that previous studies (Marwala, 2010) reveal that Particle Swarm Optimizers (PSO) outperform other algorithms like Nelder-Mead (NM) or Simulated Annealing (SA). More recent studies (Jiménez-Alonso et al., 2017) show that the HS optimizer outperforms both GA and PSO when addressing the finite element model updating of civil structures. For this reason, our proposal selects HS as global optimizer and hybridizes it with the local UKF algorithm, in order to speed up the updating process.

In order to make this paper as self-contained as possible, the three mentioned computational algorithms considering both single-objective and multi-objective approaches are next described. First, a general overview of GA is included. Next, the HS algorithm is summarized. Subsequently, the theoretical formulation of the UKF algorithm is presented. Finally, the newly proposed hybrid UKF-HS algorithm is described in detail.

\subsection{Genetic Algorithms (GA)}

Genetic algorithms (GA) are nature-inspired computational algorithms based on the natural selection theory. Thus, the assessment of each chromosome (physical parameter vector) is based on the value of the objective function for each candidate.

The GA, under the single-objective approach, can be summarized in the following steps (Koh and Perry, 2009): (i) an initial population of parameter vectors is randomly created; (ii) the objective function is evaluated for all the candidates; (iii) an iterative process is developed where parameter vectors are selected in order to create a new population; (iv) a new population is created using both mechanisms, the crossover, which allows obtaining a new vector from to previous ones, and the mutation, which consists in modifying the value of one component of the parameter vector to explore new areas of the search domain; (v) the objective function is evaluated for the new population at each iteration and the candidates with higher fitness are selected; (iv) the 
steps (iii) to (v) are repeated iteratively until a convergence criterion is met. The flowchart of the GA is shown in Figure 1a.

On the other hand, the genetic algorithms, under the multi-objective approach (MGA), are addressed by the non-dominated sorting genetic algorithms (the so-called NSGA-II algorithm) proposed by Deb et al. (2002). According to this algorithm, the non-dominated solutions are classified using the non-dominated sorting method. In this manner, a new population is generated considering only the non-dominated solutions. Both mechanisms are controlled by an operator denominated crowded comparison operator. This operator is based on two attributes of each solution: (i) the rank; and (ii) the crowding distance. As result, a set of solutions, which constitutes the Pareto front, is obtained. The flowchart of the MGA algorithm is shown in Figure $1 \mathrm{~b}$.

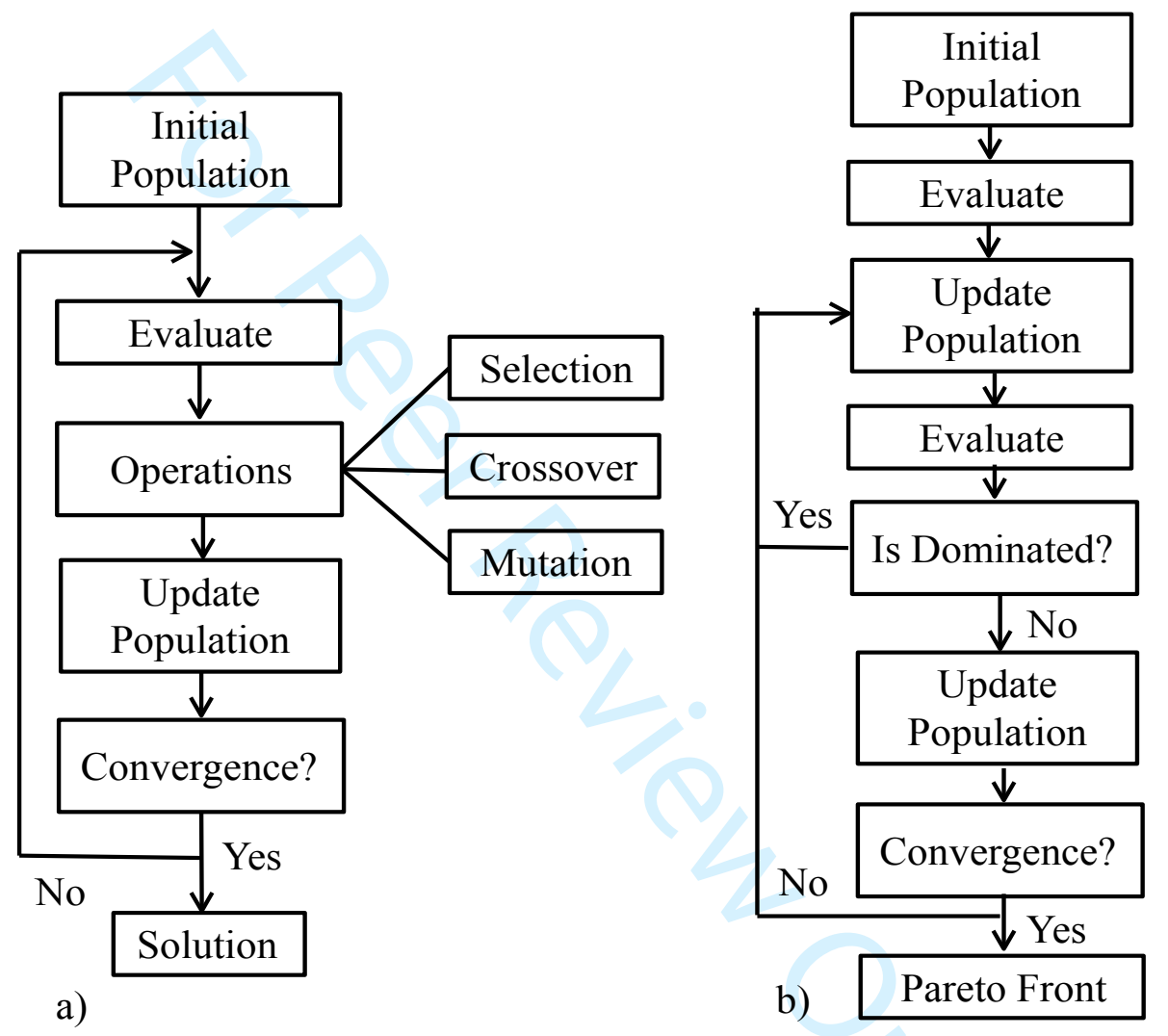

Figure 1. Flowchart of the genetic algorithms: a) GA and b) MGA.

\subsection{Harmony Search Algorithm (HS)}

The HS algorithm was proposed by Geem et al. (2001). The algorithm is inspired in the musical improvisation where harmony is searched according to aesthetic requirements. As the GA, the HS finds the global minimum of the objective function by modifying iteratively the value of the considered physical parameters of the model. Although this algorithm has been implemented successfully for several practical engineering applications (Manjarres et al., 2013); however it has not been yet implemented for the finite-element-model updating of civil engineering structures according to the best of the authors' knowledge.

The HS algorithm, under the single-objective approach, can be summarized in the following steps: (i) the harmony matrix, $\mathbf{H}$, is created (which contains the initial candidate solutions, parameter vector, created randomly); (ii) the objective function is evaluated for each solution; (iii) a new harmony is generated using three different mechanisms (memory consideration, pitch adjustments and randomization); (iv) the 
objective function is evaluated for each new harmony, (v) the harmony matrix, $\mathbf{H}$, is updated with the best generated harmonies; and (vi) the steps (iii) to (v) are repeated until a convergence criterion is met.

When a new harmony is generated, each element of a new candidate vector can be defined in terms of either a previous value stored in the harmony matrix, $\mathbf{H}$, or adopting a new random value. This fact is controlled by the harmony memory consideration rate, $H M C R$. This ratio establishes the probability of selecting a previous component of the harmony matrix, H. Additionally, when some elements adopts the value of a previous one, it can be mutated according to the pitch adjustment rate, PAR. This ratio establishes the probability of mutating an element of the candidate vector. The adjustment is based on an additional parameter, the so-called bandwidth, $b w$, which is added or subtracted to mutate the considered candidate vector. The flowchart of the HS algorithm is shown in Figure 2a.

The HS algorithm, under the multi-objective approach (MHS), is an extension of the above mentioned algorithm which allows minimizing multi-objective functions. At each iteration, a set of new possible candidate solutions is created according to both the $H M C R$ and $P A R$ ratios. The classification of the non-dominated solutions is performed using both the non-dominated sorting method (Deb et al., 2002) and the crowding distance. In order to restore the initial size of the harmony matrix, $\mathbf{H}$, the worst solutions in terms of crowding distance are deleted. These steps are repeated iteratively until a convergence criterion is met. As result of this process, a set of possible solutions is obtained, the so-called Pareto front. The flowchart of the MHS algorithm is shown in Figure $2 b$.

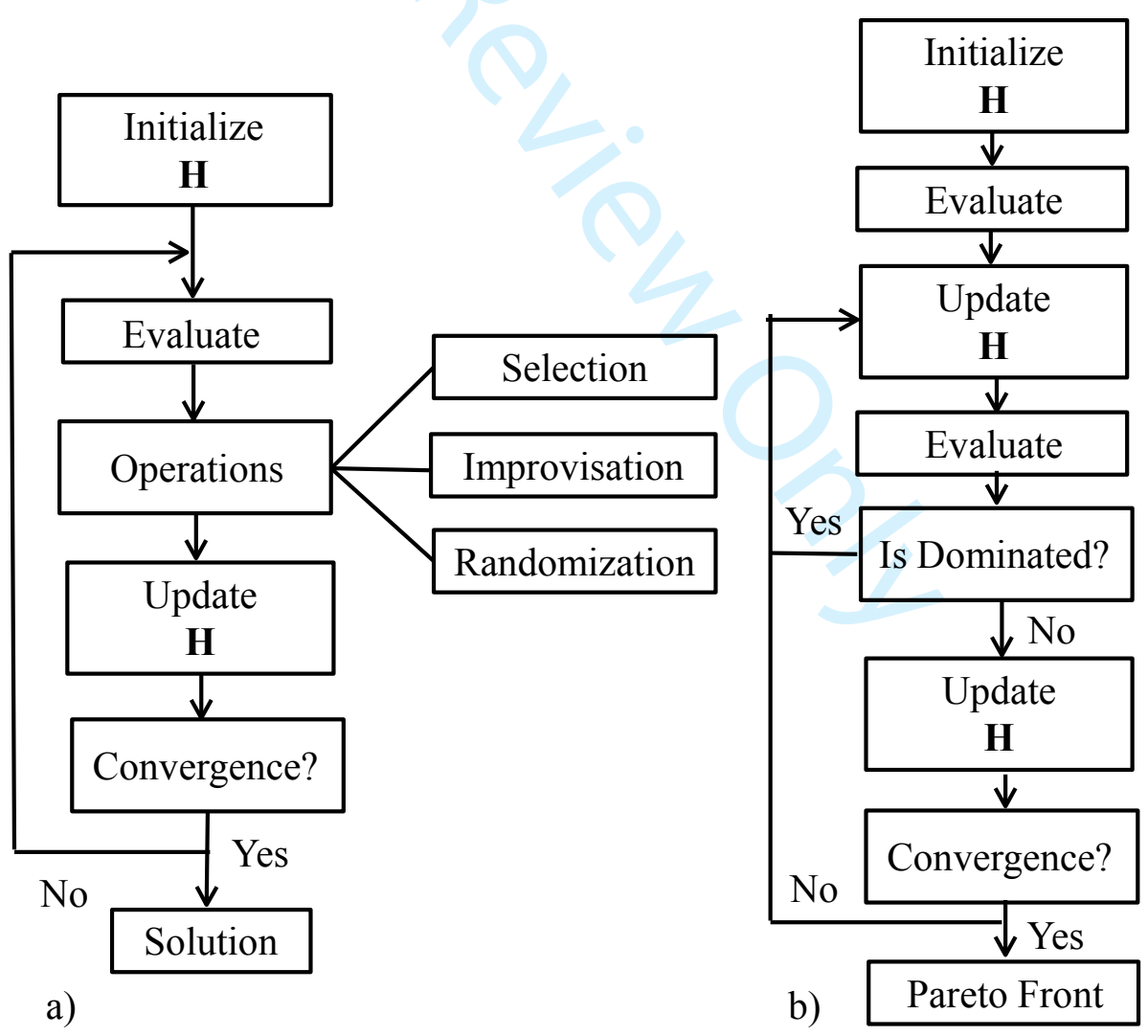

Figure 2. Flowchart of the harmony search algorithm: a) HS and b) MHS.

\subsection{Unscented Kalman Filter (UKF)}


A local minimization algorithm has been combined with the HS algorithm to reduce the simulation time needed to perform the updating process. The unscented Kalman filter (UKF) has been selected for this purpose (Julier and Uhlmann, 1997). This algorithm is a derivative free estimator, which involves that neither Jacobians nor Hessians must be computed, widely used for the state-estimation of nonlinear dynamic systems. Among the different transformations, the scaled unscented transformation proposed by Wan and Van Der Merwe (2000) has been adopted herein to define the UKF algorithm.

The formulation for a parameter identification problem may be represented as follows (Wan et al., 2000):

$$
\begin{gathered}
\boldsymbol{\theta}_{k}=\boldsymbol{\theta}_{k-1}+\mathbf{w}_{k-1} \\
\mathbf{z}_{k}=h\left(\boldsymbol{\theta}_{k}\right)+\mathbf{v}_{k}
\end{gathered}
$$

where $\boldsymbol{\theta}$ is the parameter vector; $h$ is the nonlinear modelling function, $\mathbf{z}$ extracts the outputs of the dynamic system; $\mathbf{w}$ is the statistical noise of the identification process; and $\mathbf{v}$ is the statistical noise of observation process. Both type of noise are assumed to be uncorrelated and white Gaussian noise with zero-mean and covariance matrices $\mathbf{Q}$ and $\mathbf{R}$ respectively. It is necessary to remark that the matrix $\mathbf{R}$ may be computed by means of two terms (Tarantola, 2005): (i) the measurement noise; and (ii) the modelling noise. As the same models have been employed during the simulation and estimation phases, the effects of modelling noise has not been considered herein (Astroza et al., 2016).

The UKF is a straightforward extension of the unscented transformation (Julier and Uhlmann, 2004). The estimation is addressed by considering $2 n_{d}+1$ (being $n_{d}$ the number of parameters) deterministic sampling points (sigma points) which can be used to evaluate the true mean and the covariance. The propagation of these sigma points through the nonlinear function $h$ leads to compute the true posterior mean and covariance of the estimated parameters up to the second order of the Taylor series expansion of the nonlinear function (third order of the Taylor series expansion for a Gaussian inputs). The determination of the sigma points is based on the square-root decomposition of the posterior covariance matrix, $\mathbf{P}$. For this reason, the matrix $\mathbf{P}$ must be positive semidefinite at each step. Even though the square-root decomposition can be efficiently derived using the Cholesky factorization $\left(\mathbf{A}=\sqrt{\mathbf{P}}=\operatorname{chol}(\mathbf{P})\right.$ being $\mathbf{P}=\mathbf{A} \mathbf{A}^{\mathrm{T}}$ ), the matrix $\mathbf{P}$ is still updated at each iteration and numerical errors can give a nonpositive semidefinite matrix $\mathbf{P}$. The square-root UKF algorithm, proposed by Van Der Merwe and Wan (2001), overcomes this issue since it avoids factorising at each step. This algorithm propagates directly the matrix $\mathbf{A}$ and guarantees that the covariance matrix is positive semi-definite.

The square-root UKF algorithm has two steps: (i) the prediction step; and (ii) the correction (update) step.

The prediction step is carried out considering the prior model to evaluate the sigma points and predict the estimates of the estimation error covariance, $\mathbf{A}^{\theta}$, and calculate the model outputs, $\boldsymbol{z}_{k \mid k-1}$. The $2 n_{d}+1$ sigma points are computed as:

$$
\begin{gathered}
\left(\chi_{k-1}\right)_{0}=\hat{\boldsymbol{\theta}}_{k-1 \mid k-1} \\
\left(\chi_{k-1}\right)_{i}=\hat{\boldsymbol{\theta}}_{k-1 \mid k-1}+\sqrt{\left(n_{d}+\lambda\right)\left(\mathbf{A}_{k-1 \mid k-1}^{\theta}\right)_{i} \quad i=1,2, \ldots, n_{d}} \\
\left(\chi_{k-1}\right)_{i+n}=\hat{\boldsymbol{\theta}}_{k-1 \mid k-1}-\sqrt{\left(n_{d}+\lambda\right)}\left(\mathbf{A}_{k-1 \mid k-1}^{\theta}\right)_{i} \quad i=1,2, \ldots, n_{d}
\end{gathered}
$$


where $\hat{\boldsymbol{\theta}}_{k-1 \mid k-1}$ are the posterior parameters estimated at the previous step and $\lambda$ is a scaling parameter. The sigma points are weighted under the assumption that the sum of all the weights must be equal to the unit. The weights are defined as:

$$
\begin{gathered}
W_{0}=\frac{\lambda}{n_{d}+\lambda} \\
W_{i}=W_{i+n}=\frac{1}{2\left(n_{d}+\lambda\right)} \quad i=1,2, \ldots, n_{d}
\end{gathered}
$$

For parameter identification problems, the estimation error covariance can be calculated by applying an exponential weighting on past data, $\mathbf{A}_{k}^{\theta}=\gamma^{-1 / 2} \mathbf{A}_{k-1}^{\theta}$, being $\gamma$ a scalar factor slightly less than the unit (Van Der Merwe and Wan, 2001).

The correction (update) step consists in estimating the posterior mean and estimation error covariance in terms of the Kalman's gain matrix, $\mathbf{K}$; the measurements, $\mathbf{z}^{o b s}$; and the model outputs.

The Kalman's gain matrix is derived from the model outputs error covariance, $\mathbf{S}^{z}$, and the cross covariance between the estimation error and the model outputs error covariances, $\mathbf{P}^{\theta z}$. In order to calculate the model outputs error covariance, $\mathbf{S}^{z}$, the following expression (considering that $W_{i}>0$ for all $i \geq 1$ ) can be used (Terejanu, 2011):

$$
\begin{array}{r}
\mathbf{S}_{k}^{Z}=\sum_{0}^{2 n_{d}} W_{i}\left[\left(z_{k \mid k-1}^{\mathrm{i}}-\hat{\mathbf{z}}_{k \mid k-1}\right) \cdot\left(\boldsymbol{z}_{k \mid k-1}^{\mathrm{i}}-\hat{\mathbf{z}}_{k \mid k-1}\right)^{T}\right]+\mathbf{R}= \\
{\left[\sqrt{W_{i}}\left(\boldsymbol{z}_{k \mid k-1}^{\mathrm{i}}-\hat{\mathbf{z}}_{k \mid k-1}\right), \sqrt{\mathbf{R}}\right] \cdot\left[\sqrt{W_{i}}\left(\boldsymbol{z}_{k \mid k-1}^{\mathrm{i}}-\hat{\mathbf{z}}_{k \mid k-1}\right)^{T}, \sqrt{\mathbf{R}^{T}}\right]^{T}+W_{0}} \\
{\left[\left(\boldsymbol{z}_{k \mid k-1}^{0}-\hat{\mathbf{z}}_{k \mid k-1}\right) \cdot\left(\boldsymbol{z}_{k \mid k-1}^{0}-\hat{\mathbf{z}}_{k \mid k-1}\right)^{T}\right] \quad \text { for } i=1: 2 n_{d}}
\end{array}
$$

The first term can be expressed by means of a $q r$ factorization $^{1}$ as the product of an orthogonal matrix $\mathbf{G}_{k} \in \mathbb{R}^{2 n_{d}+r \times r}$ and an upper triangular matrix $\mathbf{S}_{k}^{Z} \in \mathbb{R}^{r \times r}$, being $r$ the number of measurements. The last term, can be taken into account performing a rank 1 update to Cholesky factorization ${ }^{2}$. Therefore, the matrix $\boldsymbol{S}_{k}^{Z}$ can be calculated as (Terejanu, 2011):

$$
\begin{gathered}
\mathbf{S}_{k}^{Z}=\operatorname{qr}\left(\left[\sqrt{W_{1: 2 n_{d}}} \cdot\left[\left(\boldsymbol{z}_{k \mid k-1}\right)_{1: 2 n_{d}}-\hat{\mathbf{z}}_{k \mid k-1}\right], \sqrt{\mathbf{R}}\right]\right) \\
\mathbf{S}_{k}^{Z}=\operatorname{cholupdate}\left(\mathbf{S}_{k}^{Z},\left(\boldsymbol{z}_{k \mid k-1}\right)_{0}-\hat{\boldsymbol{z}}_{k \mid k-1}, \operatorname{sgn}\left(W_{0}\right) W_{0}\right)
\end{gathered}
$$

Once the posterior mean of the parameter estimates, $\hat{\boldsymbol{\theta}}_{\boldsymbol{k} \mid \boldsymbol{k}}$ and the posterior estimation error covariance, $\mathbf{A}_{k \mid k}^{\theta}$, are obtained, the algorithm follows a iterative process until the maximum number of iterations is reached.

The considered algorithm may be formulated as follows.

Initial Step:

$$
\hat{\boldsymbol{\theta}}_{0}=\boldsymbol{\theta}_{\text {prior }}
$$

\footnotetext{
${ }^{1}$ The $q r$ factorization of a matrix $\mathbf{M}$ allows expressing the matrix as $\mathbf{M}=\mathbf{Q R}$. Here, $\mathbf{M}$ is an $m$ by- $n$ matrix, $\mathbf{R}$ is an $m$-by- $n$ upper triangular matrix and $\mathbf{Q}$ is an $m$-by- $m$ unitary matrix. ${ }^{2}$ If $\mathbf{R}=\operatorname{chol}(\mathbf{A})$, the Cholesky factor of the rank 1 update $\mathbf{A}+\mathbf{v v}^{\mathrm{T}}$ is written as $\mathbf{S}$ $=$ cholupdate $(\mathbf{R}, \mathbf{v})$.
} 


$$
\mathbf{A}_{0}^{\theta}=\operatorname{chol}\left(\mathbf{P}_{0}^{\theta}\right)
$$

Main loop: for $k=1: N_{U K F}$ (number of iterations of the UKF)

Prediction step:

$$
\begin{aligned}
& \text { Calculate the } 2 n_{d}+1 \text { Sigma Points: }\left(\chi_{k-1}\right)_{i} \\
& \qquad \begin{array}{c}
\left(\chi_{k \mid k-1}\right)_{i}=\left(\chi_{k-1}\right)_{i} \\
\boldsymbol{\theta}_{k \mid k-1}=\sum_{0}^{2 n_{d}} W_{i} \cdot\left(\chi_{k \mid k-1}\right)_{i} \\
\mathbf{A}_{k \mid k-1}^{\theta}=\gamma^{-\frac{1}{2}} \mathbf{A}_{k-1}^{\theta} \\
\left(\boldsymbol{z}_{k \mid k-1}\right)_{i}=\boldsymbol{h}\left(\left(\boldsymbol{\chi}_{k \mid k-1}\right)_{i}\right) \\
\hat{\mathbf{z}}_{k \mid k-1}=\sum_{0}^{2 n_{d}} W_{i} \cdot\left(\boldsymbol{z}_{k \mid k-1}\right)_{i}
\end{array}
\end{aligned}
$$

Correction (update) step:

$$
\begin{gathered}
\mathbf{S}_{k \mid k-1}^{Z}=\operatorname{qr}\left(\left[\sqrt{W_{1: 2 n_{d}}} \cdot\left[\left(\boldsymbol{z}_{k \mid k-1}\right)_{1: 2 n_{d}}-\hat{\mathbf{z}}_{k \mid k-1}\right] \sqrt{\mathbf{R}}\right]\right) \\
\mathbf{S}_{k \mid k-1}^{Z}=\text { cholupdate }\left(\mathbf{S}_{k \mid k-1}^{Z},\left(\boldsymbol{z}_{k \mid k-1}\right)_{0}-\hat{\mathbf{z}}_{k \mid k-1}, \operatorname{sgn}\left(W_{0}\right)\right) \\
\mathbf{P}_{k \mid k-1}^{\theta Z}=\sum_{0}^{2 n_{d}}\left(W_{i}\left[\left(\boldsymbol{\chi}_{k \mid k-1}\right)_{i}-\hat{\boldsymbol{\theta}}_{k \mid k-1}\right] \cdot\left[\left(\boldsymbol{z}_{k \mid k-1}\right)_{i}-\hat{\mathbf{z}}_{k \mid k-1}\right]^{T}\right) \\
\mathbf{K}_{k}=\left(\mathbf{P}_{k \mid k-1}^{\theta z} / \mathbf{S}_{k \mid k-1}^{Z}\right) / \mathbf{S}_{k \mid k-1}^{z} \\
\hat{\boldsymbol{\theta}}_{\boldsymbol{k} \mid \boldsymbol{k}}=\hat{\boldsymbol{\theta}}_{k \mid k-1}+\mathbf{K}_{k}\left(\mathbf{z}^{o b s}-\hat{\mathbf{z}}_{k \mid k-1}\right) \\
\mathbf{U}=\mathbf{K}_{k} \mathbf{S}_{k \mid k-1}^{Z} \\
\mathbf{A}_{k \mid k}^{\theta}=\text { cholupdate }\left(\mathbf{A}_{k \mid k-1}^{\theta}, \mathbf{U},-1\right)
\end{gathered}
$$

end

\subsection{Hybrid UKF-HS algorithm}

The local minimization procedure followed by the square root UKF is implemented in both the HS and the MHS algorithms to reduce the simulation time required to solve the finite-element-model updating problem. The proposed hybrid algorithm is a local-global optimization algorithm which combines the main virtues of the two component algorithms. In this sense, it should be emphasized that this proposed algorithm is derivative free; not being required the computation of the Jacobian. Besides it guarantees the positive semi-definiteness of the covariance matrix as the intrinsic properties of the square root UKF algorithm are preserved.

The general layout of this new hybrid algorithm follows the general scheme of the above mentioned HS algorithm. Thus, the different steps that configure both the single-objective approach (UKF-HS) and the multi-objective approach (UKF-MHS algorithm) have been included herein. First, the new hybrid UKF-HS algorithm consists of the following steps: (i) the HS algorithm creates the initial candidate solution (parameter vector); (ii) the square-root UKF algorithm computes the mean of the parameters vector, $\boldsymbol{\theta}_{k \mid k}$; (iii) the matrix $\mathbf{H}$ is updated; (iv) the steps (ii) to (ii) are repeated until some convergence criterion is met; and (v) finally, the solution is obtained.. Thus, the flowchart of the proposed hybrid UFK-HS algorithm is shown in Figure $3 \mathrm{a}$. 
Similarly, the new hybrid UKF-MHS algorithm consists of the following steps; (i) the MHS algorithm creates the initial candidate solutions (parameter vectors); (ii) the square-root UKF algorithm gives the mean of the parameter vector, $\boldsymbol{\theta}_{k \mid k}$; (iii) the objective function is evaluated for each vector $\boldsymbol{\theta}_{k \mid k}$; (iv) the non-dominating sorting method classifies the solutions in order to delete the dominated solutions; (v) the initial size of the matrix $\mathbf{H}$ is preserved at each iteration by means of the crowding distance; (vi) the steps (ii) to (v) are repeated until some convergence criterion is met; and (vii) finally, the Pareto front is obtained. Finally, the flowchart of the proposed hybrid UFKMHS algorithm is shown in Figure $3 b$.

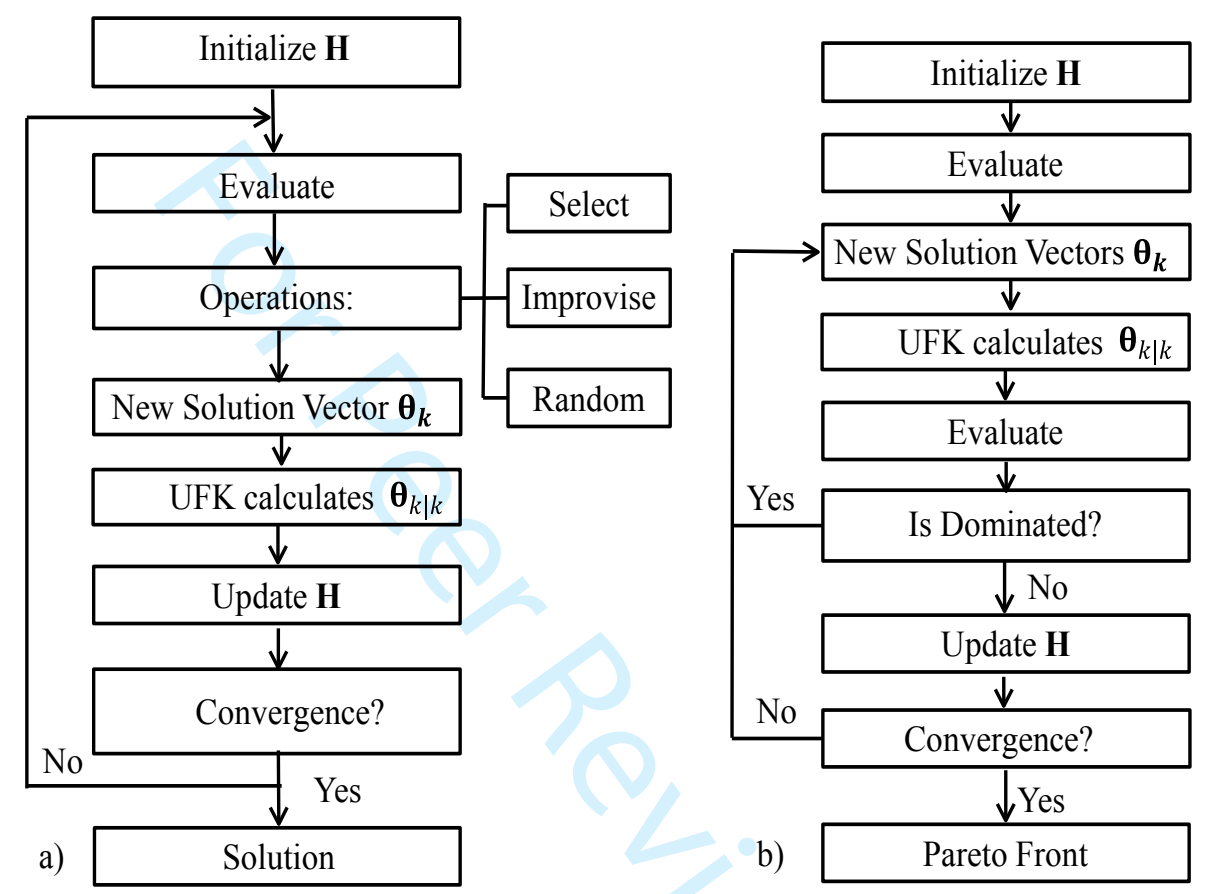

Figure 3. Flowchart of the new proposed hybrid algorithm: a) UKF-HS and b) UKFMHS.

\section{Application Example: Finite-element-model Updating of a Laboratory}

\section{Footbridge.}

In order to analyse the performance this new hybrid algorithm, when it is employed for the finite-element-model updating of civil engineering structures, a real case-study was studied. The finite-element-model updating of a real structure was performed for this purpose. As benchmark, a reconfigurable steel footbridge located at the laboratory of the Vibration Engineering Section of the University of Exeter (U.K.) was considered herein (Hudson and Reynolds, 2017). The updating problem was solved considering two approaches (single-objective and multi-objective) and three different computational algorithms (the conventional GA, HS and the new hybrid UKF-HS). Subsequently, the results obtained after the updating process are compared in order to assess the performance of each considered algorithm. Both the updating and comparison process are presented in detail in this section.

\subsection{Description of the Structure and Preliminary Finite-element-model}

The laboratory footbridge is a steel frame structure which consists of: (i) two lateral steel beams of $15 \mathrm{~m}$ of length; (ii) rectangular plates of $200 \times 12 \mathrm{~mm}$ separated longitudinally $1.25 \mathrm{~m}$; (iii) composite SPS panel (SPS, 2019) connected (bolts) to the 
lateral and transversal elements and (iv) four steel columns. The two lateral beams, separated transversally $2.5 \mathrm{~m}$, are connected to steel columns which are pinned to the ground. An overall view of the structure is illustrated in Figure 4. A more detailed description of this structure can be found in literature (Hudson and Reynolds, 2017).

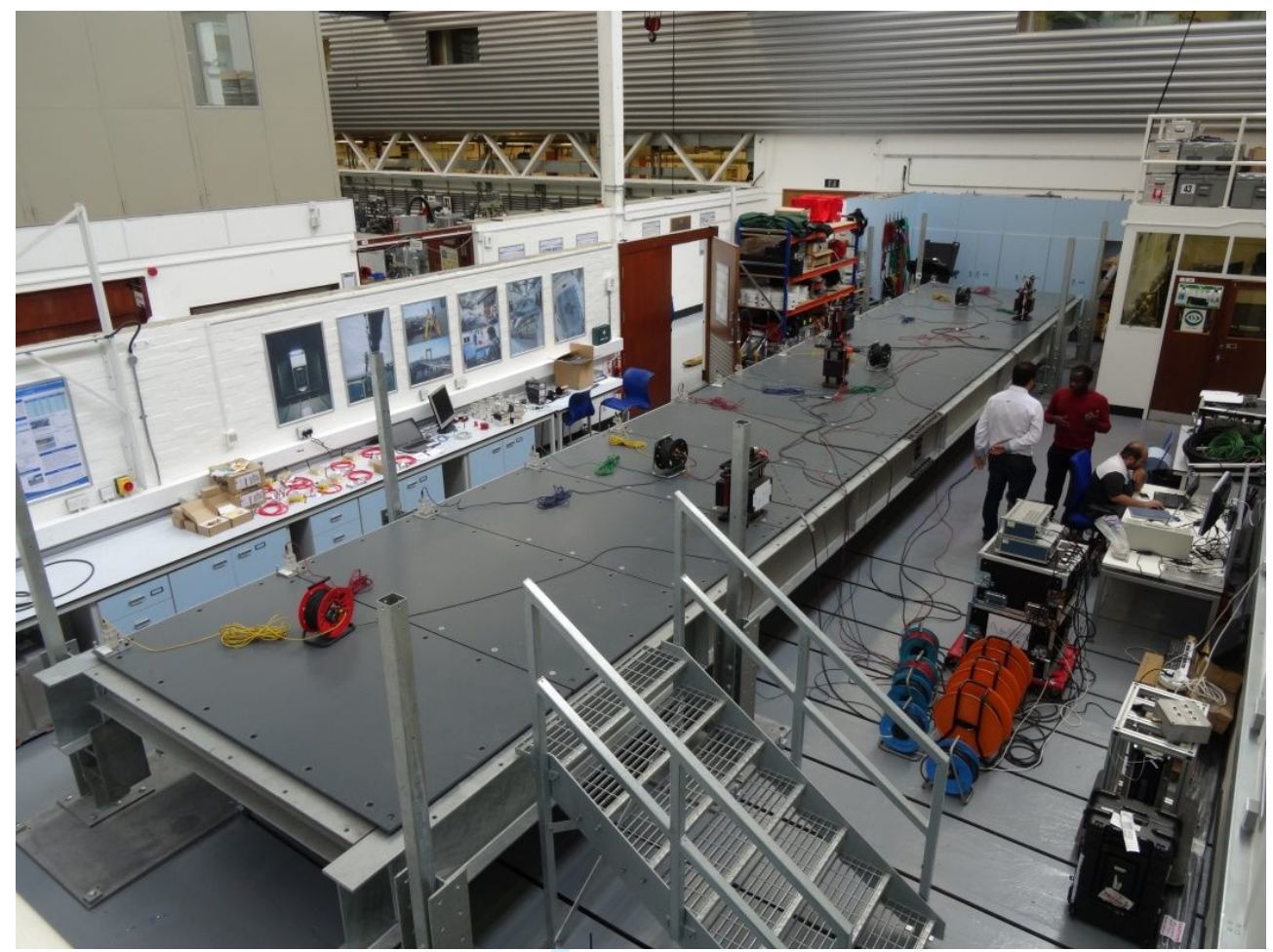

Figure 4. The reconfigurable steel footbridge located at the laboratory of the Vibration Engineering Section of the University of Exeter.

A preliminary finite-element-model of the structure was built in the software package Ansys (Ansys, 2019). A laptop computer with a $3.6 \mathrm{GHz}$ processor and a RAM memory of $8 \mathrm{~GB}$ was used for all the numerical simulations. Three different types of elements were considered for this purpose. The two lateral steel beams, the transversal steel beams and the SPS panel where modelled using 3D shell elements (SHELL181). This element has four nodes and six degrees of freedom per node. For the SPS panel, the sandwich behaviour is considered via the first-order shear-deformation theory. The bolts that connect the SPS panel and the steel structure are modelled with 3D beam elements (BEAM188). These beam elements are characterised by two nodes and six degrees of freedom per node. Finally, each support was modelled by an equivalent spring element (COMBIN14) in the longitudinal and lateral direction. An equivalent stiffness of $5.5 \cdot 10^{7} \mathrm{~N} / \mathrm{m}$ was considered for the longitudinal springs and an equivalent stiffness of $1.0 \cdot 10^{7} \mathrm{~N} / \mathrm{m}$. was taken into account for the lateral springs. Additionally, the vertical displacement of each support was constrained. The mechanical properties of the constitutive materials considered in this study were: (i) for the steel (Eurocode 3, 2005), the density, $\gamma_{s}=7850 \mathrm{~kg} / \mathrm{m}^{3}$, the Young's modulus, $E_{s}=2.1 \cdot 10^{11} \mathrm{~N} / \mathrm{m}^{2}$ and the Poisson's ratio, $v_{s}=0.3$; and (ii) for the polyurethane (Clarke, 1996), the density, $\gamma_{p}=1100 \mathrm{~kg} / \mathrm{m}^{3}$, the Young's modulus, $E_{p}=7.5 \cdot 10^{8} \mathrm{~N} / \mathrm{m}^{2}$, and the Poisson's ratio, $v_{p}=0.5$. The finite-element-model of the laboratory footbridge is shown in Figure 5. 
Figure 5. Finite-element-model, forced vibration test arrangement and considered physical parameters of the laboratory footbridge (Hudson and Reynolds, 2017).

A numerical modal analysis was performed to obtain the numerical natural frequencies and vibration modes. As result of this analysis, Table 1 shows the first seven numerical natural frequencies, $f_{n u m, j}$ (being $j$ the considered vibration mode), of the laboratory footbridge. Additionally, Figure 6 illustrates the first seven numerical vibration modes, $\phi_{\text {num }, j}$, of this structure.

\subsection{Forced Vibration Test and Experimental Modal Analysis}

In order to identify experimentally the modal properties of the footbridge, a forced vibration test was conducted. For this purpose, a set of proof-mass actuators and rowing accelerometers were employed (Figure 5). A Multiple Input-Multiple-Output (MIMO) configuration (Maia and Silva, 1997) was considered to simultaneously drive the actuators with uncorrelated random signals. The Frequency Response Functions (FRFs) for the instrumented points are derived with a $50 \%$ overlap. Subsequently, a complex mode indicator function was used to identify probable mode locations in the FRFs fitted curves. Finally, a global polynomial curve fitting method identifies the experimental natural frequencies and their vibration modes from the previous probable locations (Maia and Silva, 1997). A more detailed description of the forced vibration test and the experimental modal analysis may be found in literature (Hudson and Reynolds, 2017).

As result of this experimental modal analysis, the first seven experimental natural frequencies, $f_{\text {exp, } j}$ (being $j$ the considered vibration mode), are shown in Table 1. The first seven experimental vibration modes, $\phi_{\text {exp }, j}$, are also illustrates in Figure 6. Additionally, the agreement between the numerical and experimental modal properties of the footbridge has been checked. Thus, Table 1 shows the relative difference, $\Delta f_{\text {exp, }, j}^{n u m}$ , and the $M A C_{\text {exp }, j}^{\text {num } j}$ ratio for each considered vibration mode $j$. 
Table 1. Numerical, $f_{\text {num }, j}$, and experimental, $f_{\text {exp }, j}$, natural frequency, relative difference, $\Delta f_{\text {exp }, j}^{n u m}$, and the $M A C_{\text {exp }, j}^{n u m}$, ratio of the considered vibration mode $j$.

\begin{tabular}{ccccc}
\hline $\begin{array}{c}\text { Mode } \\
(j)\end{array}$ & $\begin{array}{c}f_{\text {num }, j} \\
{[\mathrm{~Hz}]}\end{array}$ & $\begin{array}{c}f_{\text {exp }, j} \\
{[\mathrm{~Hz}]}\end{array}$ & $\begin{array}{c}\Delta f_{\text {exp }, j}^{\text {num } j} \\
{[\%]}\end{array}$ & $\begin{array}{c}M A C_{\text {exp }, j}^{\text {num } j} \\
{[-]}\end{array}$ \\
\hline 1 & 3.652 & 3.810 & -4.147 & 0.998 \\
2 & 5.317 & 5.144 & 3.363 & 0.994 \\
3 & 8.990 & 8.485 & 5.952 & 0.988 \\
4 & 11.433 & 12.366 & -7.545 & 0.877 \\
5 & 17.795 & 18.605 & -4.354 & 0.986 \\
6 & 19.519 & 20.459 & -4.595 & 0.993 \\
7 & 20.725 & 22.980 & -9.813 & 0.634
\end{tabular}

As Table 1 shows there are some vibration modes in which the relative differences, $\Delta f_{\text {exp,j }}^{n u m}$, are greater than $5 \%$ and the $M A C_{\text {exp,j }}^{n u m}$ ratios are lower than 0.90 (Živanović et al., 2007). Thus, this preliminary finite-element-model can be improved to better reflect the real behaviour of this laboratory footbridge. A finite-element-model updating was performed for this purpose. The updating process was implemented considering two approaches (single-objective and multi-objective) and the three mentioned computational intelligence algorithms (GA, HS and hybrid UKF-HS). This updating process is described in detail in next sub-sections. 


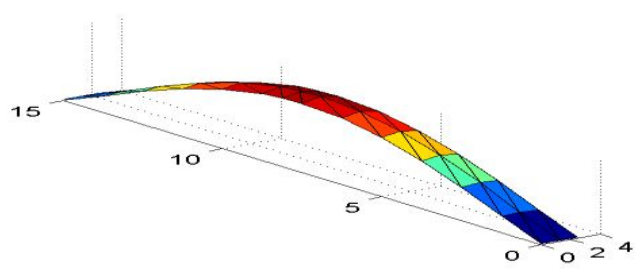

$f_{\text {num }, 1}=3.652 \mathrm{~Hz}$

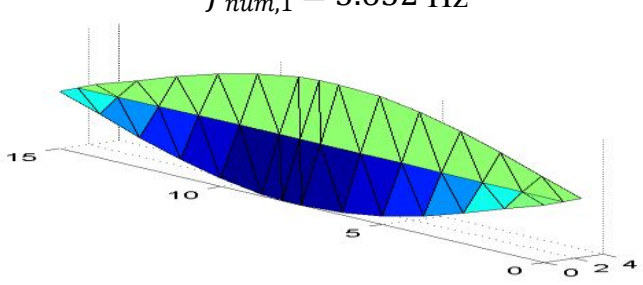

$f_{\text {num }, 2}=5.317 \mathrm{~Hz}$

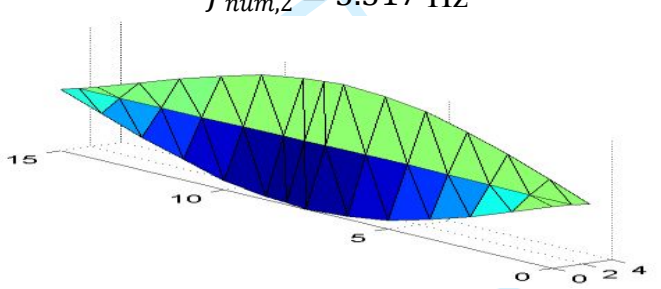

$f_{\text {num }, 3}=8.990 \mathrm{~Hz}$

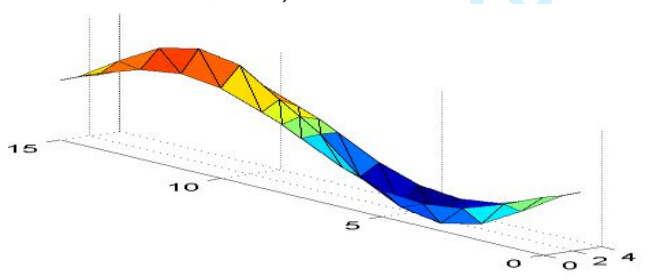

$f_{\text {num }, 4}=11.433 \mathrm{~Hz}$

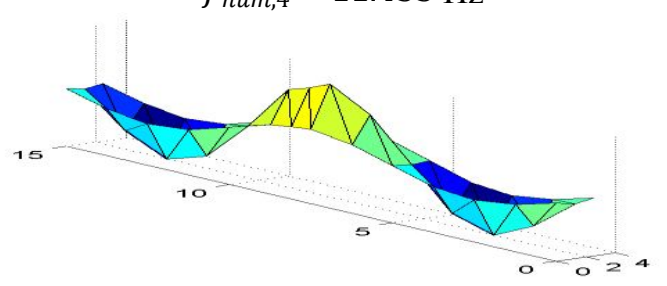

$f_{\text {num }, 5}=17.795 \mathrm{~Hz}$

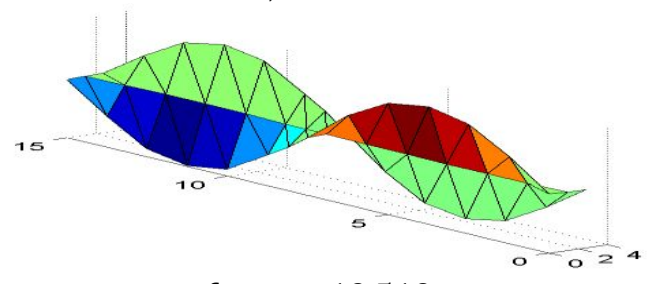

$f_{\text {num }, 6}=19.519 \mathrm{~Hz}$

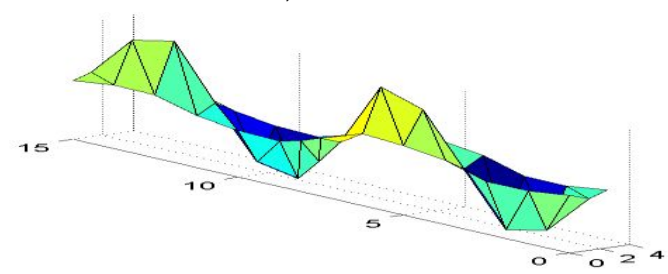

$f_{\text {num }, 7}=20.725 \mathrm{~Hz}$

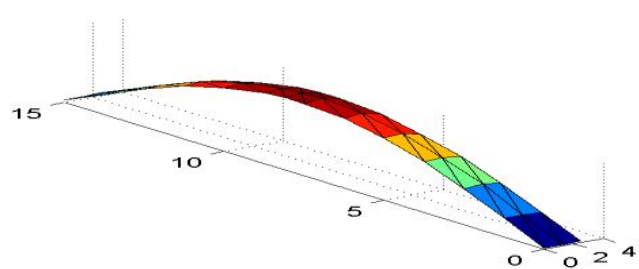

$f_{\text {exp }, 1}=3.810 \mathrm{~Hz}$

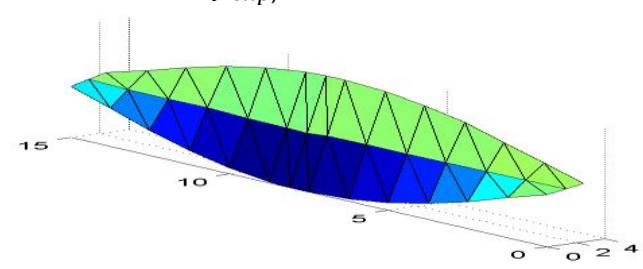

$f_{\text {exp }, 2}=5.144 \mathrm{~Hz}$

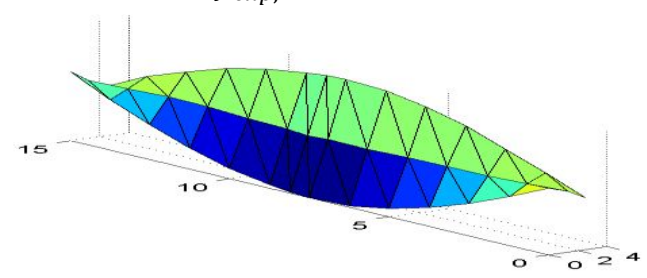

$f_{\text {exp }, 3}=8.485 \mathrm{~Hz}$

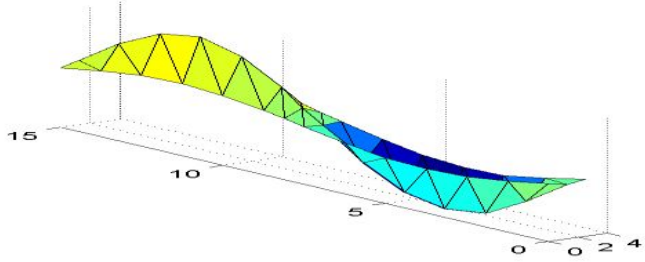

$f_{\text {exp }, 4}=12.366 \mathrm{~Hz}$

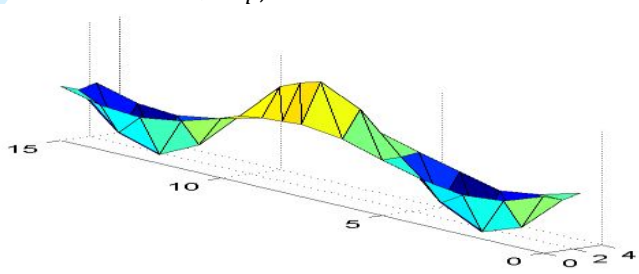

$f_{\text {exp }, 5}=18.605 \mathrm{~Hz}$

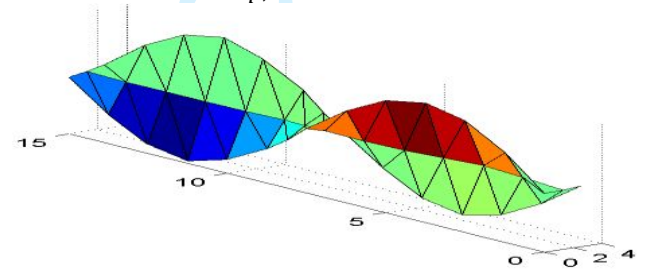

$f_{\text {exp }, 6}=20.459 \mathrm{~Hz}$

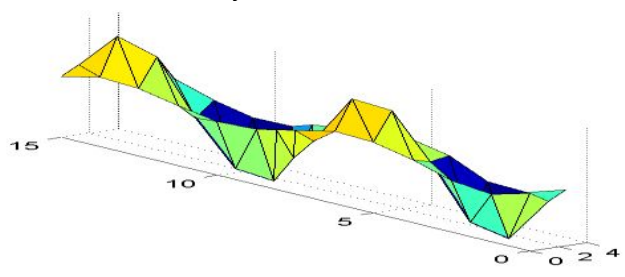

$f_{\text {exp }, 7}=22.980 \mathrm{~Hz}$

Figure 6. First seven numerical, $\phi_{\text {num }, j}$, and experimental, $\phi_{\text {exp }, j}$, vibration modes of the laboratory footbridge (being $j$ the considered vibration mode). 


\subsection{Parameters and Search Domain of the Finite-element-model Updating Process.}

As first step of this updating process, a sensitivity analysis was performed to select the most relevant physical parameters of the model. For this purpose, an initial set of fifteen physical parameters, $\boldsymbol{\theta}_{\mathbf{i n}}$, have been considered for this purpose. The initial set of physical parameters consists of: (i) the Young's modulus of the steel of the longitudinal beams in different sections, $\theta_{i n, 1}-\theta_{i n, 6}\left[\mathrm{~N} / \mathrm{m}^{2}\right]$; (ii) the Young's modulus of the steel of the SPS panel, $\theta_{i n, 7}\left[\mathrm{~N} / \mathrm{m}^{2}\right]$; (iii) the Young's modulus of the polyurethane of the SPS panel, $\theta_{i n, 8}\left[\mathrm{~N} / \mathrm{m}^{2}\right]$; (iv) the Young's modulus of the steel of the bolts, $\theta_{i n, 9}\left[\mathrm{~N} / \mathrm{m}^{2}\right]$; (v) the Young's modulus of the steel of the lateral beams, $\theta_{i n, 10}$ $\left[\mathrm{N} / \mathrm{m}^{2}\right]$; (vi) the equivalent longitudinal stiffness of each support, $\theta_{i n, 11}[\mathrm{~N} / \mathrm{m}]$; (vii) the equivalent lateral stiffness of each support, $\theta_{i n, 12}[\mathrm{~N} / \mathrm{m}]$; (viii) the equivalent vertical stiffness of each support, $\theta_{i n, 13}[\mathrm{~N} / \mathrm{m}]$; (ix) the equivalent rotational stiffness (x-axis) of each support, $\theta_{i n, 14}[\mathrm{~N} / \mathrm{rad}]$; and (x) the equivalent rotational stiffness (y-axis) of each support, $\theta_{i n, 15}[\mathrm{~N} / \mathrm{rad}]$. The selection of this initial set of parameters assumed a symmetric behaviour of the structure.

Subsequently, as the modal strain energy associated with each physical parameter is an indicator of its influence on the variation of the natural frequencies (Fox and Kapoor, 1968), this energy has been considered as basis to establish a selection criterion. For this purpose, the ratio between the modal strain energy associated with each parameter and the overall modal strain energy of the structure has been computed. A sensitivity matrix, which represents the value of the modal strain energy ratio in terms of the considered physical parameter and vibration mode, was determined. Figure 7 shows a bar graph of this sensitivity matrix. The analysis of this sensitivity matrix allows determining the most relevant physical parameters of the model (those parameters that present a significant model strain energy ratio).

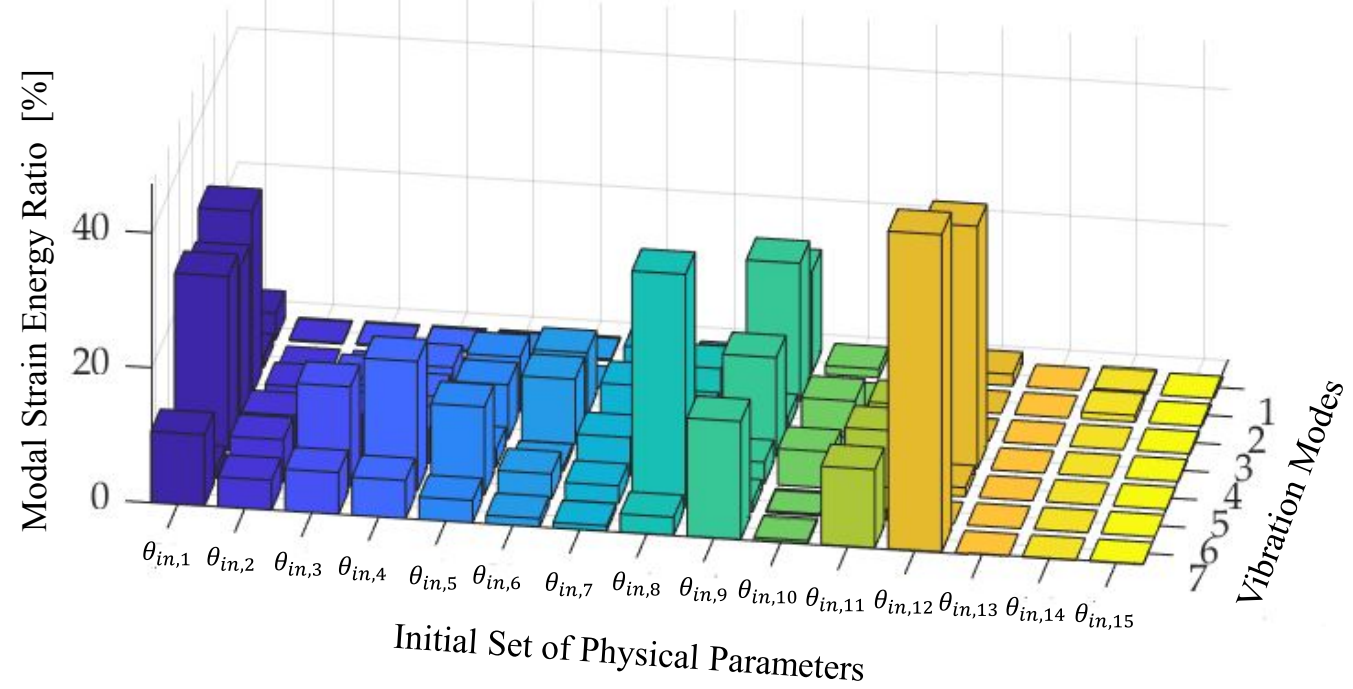

Figure 7. Sensitivity analysis in order to select the most relevant physical parameters of the model (being $\boldsymbol{\theta}_{\text {in }}=\left[\theta_{i n, 1}, \ldots, \theta_{i n, 15}\right]$ the initial set of physical parameters).

As result of this analysis, the most relevant physical parameters of this model were (Figure 5) $\boldsymbol{\theta}=\left[\theta_{1}, \theta_{2}, \theta_{3}, \theta_{4}, \theta_{5}, \theta_{6}, \theta_{7}, \theta_{8}, \theta_{9}, \theta_{10}\right]$ : (i) the Young's modulus of the steel of the longitudinal beams in different sections (Figure 5), $\theta_{1}-\theta_{6}\left[\mathrm{~N} / \mathrm{m}^{2}\right]$; (ii) the Young's modulus of the polyurethane, $\theta_{7}\left[\mathrm{~N} / \mathrm{m}^{2}\right]$; (iii) the Young's modulus of the steel 
of the bolts, $\theta_{8}\left[\mathrm{~N} / \mathrm{m}^{2}\right]$; (iv) the equivalent longitudinal stiffness of each support, $\theta_{9}$ $[\mathrm{N} / \mathrm{m}]$; and $(\mathrm{v})$ the equivalent lateral stiffness of each support, $\theta_{10}[\mathrm{~N} / \mathrm{m}]$. Additionally a search domain was established to guarantee an adequate physical meaning of the updated value of the physical parameters. The lower, $\boldsymbol{\theta}_{l}$, and upper, $\boldsymbol{\theta}_{u}$, bounds of this search domain were defined as follows; $\boldsymbol{\theta}_{l}=\left[1.9 \cdot 10^{11}, 1.9 \cdot 10^{11}, 1.9 \cdot 10^{11}, 1.9 \cdot 10^{11}\right.$ $\left., 1.9 \cdot 10^{11}, 1.9 \cdot 10^{11}, 7.5 \cdot 10^{8}, 2.1 \cdot 10^{11}, 1.4 \cdot 10^{7}, 4.8 \cdot 10^{6}\right]$ and $\boldsymbol{\theta}_{u}=\left[2.3 \cdot 10^{11}, 2.3 \cdot\right.$ $\left.10^{11}, 2.3 \cdot 10^{11}, 2.3 \cdot 10^{11}, 2.3 \cdot 10^{11}, 2.3 \cdot 10^{11}, 1.5 \cdot 10^{9}, 2.1 \cdot 10^{12}, 1.1 \cdot 10^{8}, 3.8 \cdot 10^{7}\right]$

Once both the physical parameters and their corresponding search domain have been established, the finite-element-model updating can be performed. Herein, this process has been carried out considering two approaches and the three mentioned computational intelligence algorithms.

\subsection{Finite-element-model Updating under the Single-Objective Approach.}

First, the finite-element-model updating of the footbridge was performed under the single-objective approach and the three mentioned computational algorithms (GA, HS and hybrid UKF-HS). The three considered algorithms were implemented in Matlab (Matlab, 2019). The main design variables considered for each computational algorithm were: (i) for the GA, a crossover ratio of 0.8 and a mutation ratio of 0.4 ; (ii) for the HS, a $H M C R$ ratio of 0.9 , a $P A R$ ratio of 0.3 and a $b w$ equals to the $1 \%$ of the search domain of each parameter; and (iii) for the UKF-HS, the same value for the HMCR ratio, the $P A R$ ratio and the $b w$ variable than the HS algorithm. These design parameters balance the ability of these computational algorithms to sweep the search domain and to reach accurately the global minimum of this search domain (Marwala, 2010; Jiménez-Alonso et al., 2017). Additionally for the UKF-HS algorithm, the following parameters were assumed: (i) the $\mathbf{d}_{o b s}$ vector is built from the experimental natural frequencies and a $7 \mathrm{x} 1$ vector filled with ones (since the residue of the vibration modes is defined from the $M A C_{\text {exp }, j}^{\text {upd } j}$ ratio and the aim is to make the value of the $M A C_{\text {exp,j }}^{u p d . j}$ as close as possible to the unit), (ii) a factor, $\lambda=0.0001$, was considered to compute the sigma points (Astroza et al., 2016); and (iii) a scalar factor, $\gamma=0.99$, was considered to update the estimation error covariance matrix (Van Der Merwe and Wan, 2001).

For the definition of the single-objective function, the weights associated with the residuals must be defined. For this purpose, a sensitivity study, where the values of the weights were varied between 0 and 1 considering a step of 0.1 (eleven cases), was performed. In each case, a value was assigned to each weight and the value of the objective function at the end of the optimization process, $f_{f}$, was obtained. The combination of weights that achieves the lower value of the objective function, $f_{f}$, was selected. The HS algorithm was considered for this analysis. As result, the following weights were considered: (i) for the residuals associated with the natural frequencies, $\sum_{j}^{7} w_{f, j}=0.7$; and (ii) for the residuals associated with the vibration modes, $\sum_{j}^{7} w_{m, j}=0.3$.

Next, a sensitivity study was performed to analyse the influence of three hyperparameters on the performance of the hybrid UKF-HS algorithm. For this purpose the following hyper-parameters were considered: (i) the number of iterations of the UKF algorithm, $N_{U K F}$; (ii) the initial estimation error covariance, $\boldsymbol{P}_{0}^{\boldsymbol{\theta}}$; and (iii) the measurement noise covariance matrix, $\mathbf{R}_{i i}$.

Seven simulations were run for this purpose. For these simulations, the number 
of iterations, Iter $=2$, and the population size, $P o p=5$, have been maintained constant; and the three considered hyper-parameters have been modified according to the following values: (i) three different values for the parameter, $N_{U K F},(1,3$ and 5); (ii) three different values for the parameter, $\boldsymbol{P}_{0}^{\boldsymbol{\theta}},\left(\operatorname{diag}\left(\left(\frac{\left.\boldsymbol{\theta}_{u}-\boldsymbol{\theta}_{l}\right)}{500}\right)^{2}\right), \operatorname{diag}\left(\left(\frac{\left(\boldsymbol{\theta}_{u}-\boldsymbol{\theta}_{l}\right)}{2000}\right)^{2}\right)\right.$ and $\operatorname{diag}\left(\left(\frac{\left.\boldsymbol{\theta}_{u}-\boldsymbol{\theta}_{l}\right)}{4000}\right)^{2}\right)$; and (iii) three different values for the parameter, $\mathbf{R}_{i i}(0.1,0.001$ and $0.0001)$.

As comparison criteria, the convergence speed and the accuracy of the adjustment (the value of the objective function, $f_{f}$ ) have been taken into account. It must be remarked that the converge speed has been considered through the analysis of two parameters: (i) the simulation time, Time; and (ii) the number of evaluations, Eval. This second parameter allows considering jointly the effect on the converge speed of three characteristic parameters: (i) the number of iterations of the global algorithm, Iter ; (ii) the population size, Pop; and (iii) the number of iterations of the UKF algorithm, $N_{U K F}$.

Table 2 shows the results of this sensitivity study. For the first hyper-parameter, $N_{U K F}$, an optimum value of $N_{U K F}=3$ is considered since, a reduced value, $N_{U K F}=1$, reduces the accuracy of the adjustment $\left(f_{f}=0.0037\right)$; and an excessive value, $N_{U K F}$ $=5$, increases the simulation time $\left(\right.$ Time $\left.=9.44 \cdot 10^{3} \mathrm{~s}\right)$. For the second hyperparameter, $\boldsymbol{P}_{0}^{\theta}$, an optimum value of $\boldsymbol{P}_{0}^{\theta}=\operatorname{diag}\left(\left(\frac{\left(\boldsymbol{\theta}_{u}-\boldsymbol{\theta}_{l}\right)}{2000}\right)^{2}\right)$ is established, since this value improves the accuracy of the adjustment $\left(f_{f}=0.0033\right)$ without compromising the simulation time (Time $=5.71 \cdot 10^{3} \mathrm{~s}$ ). Finally, for the third hyper-parameter, $\mathbf{R}_{i i}$, an optimum value of $\mathbf{R}_{i i}=0.001$ is considered, since this value improves the accuracy of the adjustment $\left(f_{f}=0.0033\right)$ without compromising the simulation time ( Time $\left.=5.71 \cdot 10^{3} \mathrm{~s}\right)$.

Table 2. Performance of the three considered algorithms to solve the updating problem under the single-objective approach (where Iter is the number of iterations of the main algorithm; Pop is the population size; $N_{U K F}$ is the number of iterations of the UKF algorithm; $\boldsymbol{P}_{0}^{\boldsymbol{\theta}}$ is the initial estimation error covariance; $\mathbf{R}_{i i}$ is the measurement noise covariance matrix; Eval is the number of evaluations of the objective function; Time is the time [s] required until a convergence criterion is met; and $f_{f}$ is the value of the objective function when the updating process has finished).

\begin{tabular}{|c|c|c|c|c|c|c|c|c|}
\hline & Iter & Pop & $N_{U K F}$ & $\boldsymbol{P}_{0}^{\boldsymbol{\theta}}$ & $\overline{\mathbf{R}_{i i}}$ & Eval & Time $[\mathrm{s}]$ & $f_{f}[-]$ \\
\hline GA & 50 & 20 & --- & --- & --- & 1020 & $9.79 \cdot 10^{3}$ & 0.0036 \\
\hline HS & 500 & 20 & --- & --- & --- & 520 & $6.64 \cdot 10^{3}$ & 0.0033 \\
\hline \multirow{3}{*}{ UKF-HS } & 2 & 5 & 1 & $\operatorname{diag}\left(\left(\frac{\left(\boldsymbol{\theta}_{u}-\boldsymbol{\theta}_{l}\right)}{2000}\right)^{2}\right)$ & 0.001 & 147 & $2.1 \cdot 10^{3}$ & 0.0037 \\
\hline & 2 & 5 & 3 & $\operatorname{diag}\left(\left(\frac{\left(\boldsymbol{\theta}_{u}-\boldsymbol{\theta}_{l}\right)}{500}\right)^{2}\right)$ & 0.001 & 441 & $5.68 \cdot 10^{3}$ & 0.0034 \\
\hline & 2 & 5 & 3 & $\operatorname{diag}\left(\left(\frac{\left(\mathbf{\sigma}_{u}-\mathbf{\nabla}_{l)}\right)}{2000}\right)\right.$ & 0.001 & 441 & $5.71 \cdot 10^{3}$ & 0.0033 \\
\hline
\end{tabular}




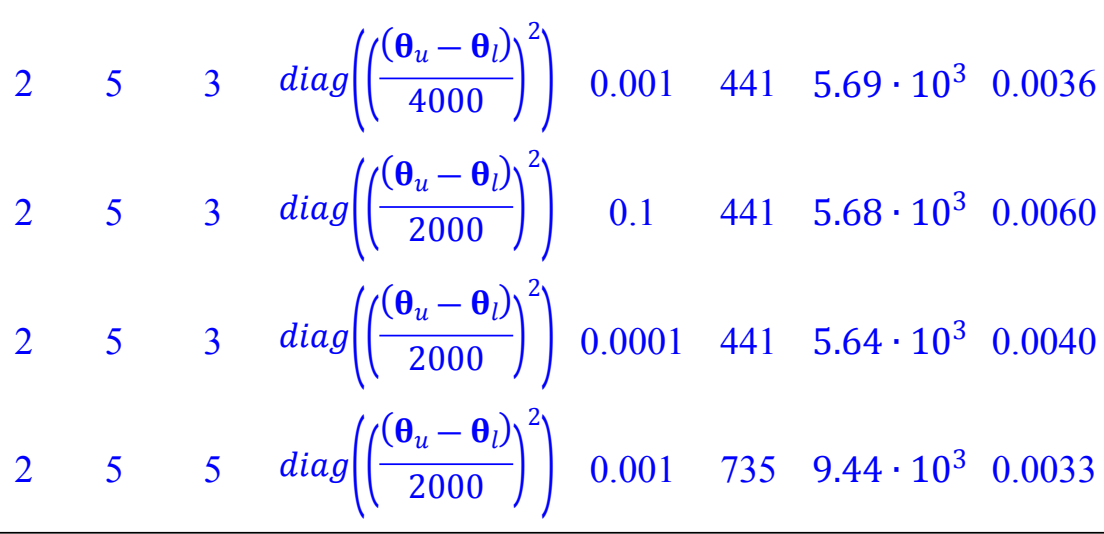

Subsequently, the performance of the updating process under the singleobjective approach for the three considered computational algorithms was analysed. As hyper-parameters of the UKF-HS algorithm, the results of the above sensitivity analysis were taken into account. Table 2 also shows the results of this performance study. As Table 2 shows the hybrid UKF-HS algorithm is clearly the most efficient algorithm, since it presents the same accuracy than the HS algorithm $\left(f_{f}=0.0033\right)$ but a lower simulation time (Time $=6.64 \cdot 10^{3} \mathrm{~s}$ for the HS against Time $=5.71 \cdot 10^{3} \mathrm{~s}$ for the hybrid UKF-HS) and number of iterations (Eval $=520$ for the HS against Eval $=441$ for the hybrid UKF-HS). Additionally, it is necessary to remark that the HS algorithm is more efficient (both convergence speed and accuracy) than the conventional GA for this optimization problem.

Additionally, Table 3 shows the updated value of the physical parameters of the model after the updating process considering the single-objective approach and the three considered computational algorithm. As Table 3 shows the correlation among the physical parameters obtained by the different algorithms is good.

Table 3. Updated value of the physical parameters of model, $\boldsymbol{\theta}$, after the updating process considering the single-objective approach and the three mentioned computational algorithms.

\begin{tabular}{cccc}
\hline $\boldsymbol{\theta}$ & GA & HS & UKF-HS \\
\hline$\theta_{1}$ & $2.29 \cdot 10^{11}$ & $2.28 \cdot 10^{11}$ & $2.29 \cdot 10^{11}$ \\
$\theta_{2}$ & $1.96 \cdot 10^{11}$ & $1.96 \cdot 10^{11}$ & $2.00 \cdot 10^{11}$ \\
$\theta_{3}$ & $2.20 \cdot 10^{11}$ & $2.15 \cdot 10^{11}$ & $2.27 \cdot 10^{11}$ \\
$\theta_{4}$ & $2.04 \cdot 10^{11}$ & $1.91 \cdot 10^{11}$ & $1.97 \cdot 10^{11}$ \\
$\theta_{5}$ & $2.08 \cdot 10^{11}$ & $2.00 \cdot 10^{11}$ & $2.11 \cdot 10^{11}$ \\
$\theta_{6}$ & $2.28 \cdot 10^{11}$ & $2.19 \cdot 10^{11}$ & $2.29 \cdot 10^{11}$ \\
$\theta_{7}$ & $1.04 \cdot 10^{9}$ & $1.28 \cdot 10^{9}$ & $1.29 \cdot 10^{9}$ \\
$\theta_{8}$ & $1.16 \cdot 10^{12}$ & $1.53 \cdot 10^{12}$ & $1.50 \cdot 10^{12}$ \\
$\theta_{9}$ & $7.67 \cdot 10^{7}$ & $7.50 \cdot 10^{7}$ & $7.50 \cdot 10^{7}$ \\
$\theta_{10}$ & $7.66 \cdot 10^{6}$ & $7.72 \cdot 10^{6}$ & $7.73 \cdot 10^{6}$ \\
\hline
\end{tabular}

Finally, Table 4 shows the updated natural frequencies, $f_{\text {upd,j, }}$, the relative differences, $\Delta f_{e x p, j}^{u p d, j}$, and the $M A C_{\text {exp }, j}^{u p d . j}$ ratio for each considered vibration mode $j$ after the updating process considering the single-objective approach and the three mentioned computational algorithms. As Table 4 shows the correlation between the experimental and numerical modal properties after the updating process is adequate for the three mentioned computational algorithms (all the relative differences, $\Delta f_{\text {exp }, j}^{u p}$, are lower than $5 \%$, and all the $M A C_{\text {exp,j }}^{u p d . j}$ ratios are greater than 0.9$)$. 
Table 4. Updated, $f_{u p d, j}$, and experimental, $f_{\text {exp }, j}$, natural frequency, relative difference, $\Delta f_{\text {exp,j }}^{u p d, j}$, and the $M A C_{\text {exp,j }}^{u p d}$ ratio for each considered vibration mode $j$ after the updating process considering the single-objective approach and the three mentioned computational algorithms.

\begin{tabular}{|c|c|c|c|c|c|c|c|c|c|c|}
\hline \multirow{2}{*}{$\begin{array}{l}\text { Mode } \\
(j)\end{array}$} & \multirow{2}{*}{$\begin{array}{l}f_{\text {exp, }} \\
{[\mathrm{Hz}]}\end{array}$} & \multicolumn{3}{|c|}{ GA } & \multicolumn{3}{|c|}{ HS } & \multicolumn{3}{|c|}{ UKF-HS } \\
\hline & & $\begin{array}{l}f_{u p d, j} \\
{[\mathrm{~Hz}]}\end{array}$ & $\begin{array}{c}\Delta f_{e x p, j}^{u p d, j} \\
{[\%]}\end{array}$ & $\begin{array}{c}M A C_{e x p, j}^{u p d . j} \\
{[-]}\end{array}$ & $\begin{array}{c}f_{u p d, j} \\
{[\mathrm{~Hz}]}\end{array}$ & $\begin{array}{c}\Delta f_{e x p, j}^{u p d, j} \\
{[\%]}\end{array}$ & $\begin{array}{c}M A C_{e x p, j}^{u p d . j} \\
{[-]}\end{array}$ & $\begin{array}{c}f_{u p d, j} \\
{[\mathrm{~Hz}]}\end{array}$ & $\begin{array}{c}\Delta f_{e x p, j}^{u p d, j} \\
{[\%]}\end{array}$ & $\begin{array}{c}M A C_{e x p, j}^{u p d . j} \\
{[-]}\end{array}$ \\
\hline 1 & 3.810 & 3.880 & 1.837 & 0.998 & 3.880 & 1.837 & 0.998 & 3.890 & 2.1 & 0.998 \\
\hline 2 & 5.144 & 5.380 & 4.588 & 0.993 & 5.372 & 4.432 & 0.993 & 5.375 & 4.491 & 0.993 \\
\hline 3 & 8.485 & 8.370 & -1.355 & 0.988 & 8.400 & -1.002 & 0.988 & 8.410 & -0.884 & 0.988 \\
\hline 4 & 12.366 & 11.930 & -3.526 & 0.907 & 11.950 & -3.364 & 0.905 & 12.002 & -2.944 & 0.903 \\
\hline 5 & 18.605 & 18.460 & -0.779 & 0.986 & 18.630 & 0.134 & 0.987 & 18.670 & 0.349 & 0.987 \\
\hline 6 & 20.459 & 20,160 & -1.461 & 0.993 & 20.150 & -1.51 & 0.992 & 20.250 & -1.022 & 0.992 \\
\hline 7 & 22.980 & 22,150 & -3.612 & 0.947 & 22.420 & -2.437 & 0.950 & 22.450 & -2.306 & 0.950 \\
\hline
\end{tabular}

In this manner, it has been validated that the hybrid UKF-HS algorithm is the most efficient algorithm among the three considered computational ones to perform the finite-element-model updating of civil engineering structure under the single-objective approach. Additionally, it has been checked that the performance of this hybrid algorithm depends on the adequate value of the three considered hyper-parameters ( $N_{U K F}, \boldsymbol{P}_{0}^{\boldsymbol{\theta}}$ and $\mathbf{R}_{i i}$ ).

\subsection{Finite-element-model Updating under the Multi-Objective Approach.}

Subsequently, the finite-element-model updating of the footbridge was performed under the multi-objective approach and the three mentioned computational algorithms (MGA, MHS and hybrid UKF-MHS). The main design variables considered for each computational algorithm were (Marwala, 2010; Jiménez-Alonso et al., 2017): (i) for the MGA, a crossover ratio of 0.7 , and a mutation ration of 0.4 ; (ii) for the MHS, a HMCR ratio of 0.9 , a $P A R$ ratio of 0.7 and a $b w$ equals to the $1 \%$ of the search domain of each parameters; and (iii) for the UKF-MHS, the same design variables than the HS algorithm (described above). The decision making problem, the selection of the best solution among the different element of the Pareto front, has been solved using the NBI method (Deb and Gupta, 2011).

As in the single-objective approach, first a sensitivity study was performed to analyse the influence of three hyper-parameters on the performance of the hybrid UKFHS algorithm. For this purpose the following hyper-parameters were considered: (i) the number of iterations of the UKF algorithm, $N_{U K F}$; (ii) the initial estimation error covariance, $\boldsymbol{P}_{0}^{\boldsymbol{\theta}}$; and (iii) the measurement noise covariance matrix, $\mathbf{R}_{i i}$. For the possible values of these hyper-parameters, the same values considered in the sensitivity analysis of the single-objective approach, were taken into account.

As comparison criteria, the convergence speed and the accuracy of the adjustment have been taken into account again. On the one hand, the converge speed has been assessed again via: (i) the simulation time, Time; and (ii) the number of evaluations, 
Eval. On the other hand, the accuracy of the adjustment has been assessed in this case via: (i) the distance between the selected point (best solution of the Pareto front) and the origin, Dist; and (ii) the sum of the two terms of the objective function, Sum.

Eight simulations were run for this purpose. On the one hand, for the analysis of the $N_{U K F}$ hyper-parameter, the number of iterations, Iter $=3$, the population size, $P o p=8$, and the new population size, New Pop $=3$, have been fixed. On the other hand, for the analysis of both $\boldsymbol{P}_{0}^{\boldsymbol{\theta}}$ and $\mathbf{R}_{i i}$ hyper-parameters, the number of iterations, Iter $=3$, the population size, $P o p=10$, and the new population size, New Pop $=3$, have also been fixed. The results of this sensitivity study are also shown in Table 3.

In relation to the $N_{U K F}$ hyper-parameter two relationships can be obtained: (i) a direct relationship between this hyper-parameter and the accuracy of the adjustment; and (ii) an indirect relationship between this hyper-parameter and the convergence speed. In relation to the $\boldsymbol{P}_{0}^{\boldsymbol{\theta}}$ hyper-parameter, a direct relationship has been obtained. Thus, a reduction of the initial estimation error covariance involves an increase of the accuracy of the adjustment without compromising the simulation time. Nevertheless, the influence of this hyper-parameter is low (as Table 5 illustrates) and an intermediate value can be considered without affecting both the goodness of the adjustment and the simulation time. In relation to the $\mathbf{R}_{i i}$ hyper-parameter, it has been checked that the intermediate value, $\mathbf{R}_{i i}=0.001$, improves the accuracy of the adjustment without compromising the simulation time.

Finally, the following values of the hyper-parameters were recommended: (i) $N_{U K F}=3$; (ii) $\boldsymbol{P}_{0}^{\boldsymbol{\theta}}=\operatorname{diag}\left(\left(\frac{\left(\boldsymbol{\theta}_{u}-\boldsymbol{\theta}_{l}\right)}{2000}\right)^{2}\right)$; and (iii) $\mathbf{R}_{i i}=0.001$. These values were considered for the subsequent performance analysis.

Table 5. Performance of the three considered algorithms to solve the updating problem under the multi-objective approach (where Iter is the number of iterations of the main algorithm; Pop is the population size; New Pop is the new population size; $N_{U K F}$ is the number of iterations of the UKF algorithm; $\boldsymbol{P}_{0}^{\boldsymbol{\theta}}$ is the initial estimation error covariance; $\mathbf{R}_{i i}$ is the measurement noise covariance matrix; Eval is the number of evaluations of the objective function; Time is the time [s] required until a convergence criterion is met; Dist is the distance between the selected point and the origin; and Sum is the sum of the two terms of the objective function).

\begin{tabular}{|c|c|c|c|c|c|c|c|c|c|c|}
\hline & $\begin{array}{c}\text { Iter } \\
.\end{array}$ & $\begin{array}{c}\text { Pop } \\
.\end{array}$ & $\begin{array}{c}\text { New Pop } \\
\text {. }\end{array}$ & $N_{U K F}$ & $P_{0}^{\theta}$ & $\mathbf{R}_{i i}$ & Eval & $\begin{array}{c}\text { Time }[\mathrm{s}] \\
x 10^{5}\end{array}$ & $\begin{array}{l}\text { Dist } \\
x 10^{4}\end{array}$ & $\begin{array}{l}\text { Sum } \\
x 10^{4}\end{array}$ \\
\hline \multirow{3}{*}{ MGA } & 25 & 50 & --- & --- & --- & --- & 1450 & 0.18 & 50.7 & 68.6 \\
\hline & 45 & 50 & --- & --- & --- & --- & 2570 & 0.33 & 50.2 & 66.9 \\
\hline & 150 & 100 & --- & --- & --- & --- & 16600 & 2.12 & 52.0 & 68.0 \\
\hline \multirow{3}{*}{ MHS } & 50 & 50 & 25 & --- & --- & --- & 1300 & 0.17 & 45.4 & 62.1 \\
\hline & 100 & 100 & 25 & --- & -- & --- & 2600 & 0.33 & 45.1 & 61.8 \\
\hline & 300 & 200 & 50 & --- & --- & --- & 15200 & 1.98 & 43.7 & 60.6 \\
\hline \multirow{3}{*}{$\begin{array}{l}\text { UKF- } \\
\text { MHS }\end{array}$} & 3 & 10 & 3 & 3 & $\operatorname{diag}\left(\left(\frac{\left(\boldsymbol{\theta}_{u}-\boldsymbol{\theta}_{l}\right)}{2000}\right)^{2}\right)$ & 0.001 & 1197 & 0.16 & 45.2 & 62.1 \\
\hline & 3 & 10 & 3 & 3 & $\operatorname{diag}\left(\left(\frac{\left(\boldsymbol{\theta}_{u}-\boldsymbol{\theta}_{l}\right)}{500}\right)^{2}\right)$ & 0.001 & 1197 & 0.15 & 46.0 & 63.5 \\
\hline & 3 & 10 & 3 & 3 & $\operatorname{diag}\left(\left(\frac{\left(\boldsymbol{\theta}_{u}-\boldsymbol{\theta}_{l}\right.}{4000}\right)^{2}\right)$ & 0.001 & 1197 & 0.15 & 44.6 & 61.4 \\
\hline
\end{tabular}




\begin{tabular}{|c|c|c|c|c|c|c|c|c|c|c|}
\hline 3 & 10 & 3 & 3 & diag & $\left.\left(\frac{\left(\boldsymbol{\theta}_{u}-\boldsymbol{\theta}_{l}\right)}{2000}\right)^{2}\right)$ & 0.1 & 1197 & 0.15 & 52.6 & 71.3 \\
\hline 3 & 10 & 3 & 3 & diag & $\left.\left(\frac{\left(\boldsymbol{\theta}_{u}-\boldsymbol{\theta}_{l}\right.}{2000}\right)^{2}\right)$ & 0.0001 & 1197 & 0.15 & 145.0 & 177.6 \\
\hline 8 & 15 & 3 & 3 & diag & $\left.\left(\frac{\left(\boldsymbol{\theta}_{u}-\boldsymbol{\theta}_{l}\right.}{2000}\right)^{2}\right)$ & 0.001 & 2457 & 0.32 & 44.3 & 61.1 \\
\hline 20 & 40 & 10 & 3 & diag & $\left.\left(\frac{\left(\boldsymbol{\theta}_{u}-\boldsymbol{\theta}_{l}\right)}{2000}\right)^{2}\right)$ & 0.001 & 15120 & 1.96 & 43.7 & 60.4 \\
\hline 3 & 8 & 3 & 1 & diag & $\left.\left(\frac{\left(\boldsymbol{\theta}_{u}-\boldsymbol{\theta}_{l}\right)}{2000}\right)^{2}\right)$ & 0.001 & 357 & 0.05 & 50.6 & 68.2 \\
\hline 3 & 8 & 3 & 3 & diag & $\left.\left(\frac{\left(\boldsymbol{\theta}_{u}-\boldsymbol{\theta}_{l}\right)}{2000}\right)^{2}\right)$ & 0.001 & 1071 & 0.14 & 49.1 & 66.5 \\
\hline 3 & 8 & 3 & 5 & diag & $\left.\left(\frac{\left(\boldsymbol{\theta}_{u}-\boldsymbol{\theta}_{l}\right.}{2000}\right)^{2}\right)$ & 0.001 & 1785 & 0.23 & 45.2 & 62.0 \\
\hline
\end{tabular}

Subsequently, the performance of the updating process under the multi-objective approach for the three considered computational algorithms was analysed. Three simulations have been performed for each computational algorithm in which several design parameters of the algorithms have been modified. The modified design variables are: (i) the number of iterations of the main algorithm, Iter; (ii) the population size, Pop; and (iii) the new population size, New Pop.

Table 5 shows the results of this performance analysis. According to these results, the hybrid UKF-MHS algorithm is again the most efficient algorithm, since it presents the same accuracy than the MHS algorithm (Dist $=43.7 \cdot 10^{4}$ and Sum $<60.6 \cdot 10^{-4}$ ) but a lower simulation time (Time $=1.96 \cdot 10^{5} \mathrm{~s}$ ) and number of iterations ( Eval = 151200). Additionally, it must be noted that the MHS algorithm is again more efficient (both convergence speed and accuracy) than the conventional MGA for this optimization problem.

Additionally, Table 6 shows the updated value of the physical parameters of the model after the updating process considering the multi-objective approach and the three considered computational algorithm. The cases with a higher number of evaluations have been shown and compared (MGA, Eval = 16600; MHS, Eval = 15200; and UKFMHS, Eval =15120). Table 6 shows the correlation among the physical parameters obtained by the different algorithms is good.

Table 6. Updated value of the physical parameters of model, $\boldsymbol{\theta}$, after the updating process considering the multi-objective approach and the three mentioned computational algorithms.

\begin{tabular}{cccc}
\hline $\boldsymbol{\theta}$ & MGA & MHS & UKF-MHS \\
\hline$\theta_{1}$ & $2.29 \cdot 10^{11}$ & $2.27 \cdot 10^{11}$ & $2.28 \cdot 10^{11}$ \\
$\theta_{2}$ & $2.15 \cdot 10^{11}$ & $2.29 \cdot 10^{11}$ & $2.26 \cdot 10^{11}$ \\
$\theta_{3}$ & $2.06 \cdot 10^{11}$ & $2.22 \cdot 10^{11}$ & $2.26 \cdot 10^{11}$ \\
$\theta_{4}$ & $2.14 \cdot 10^{11}$ & $2.22 \cdot 10^{11}$ & $2.28 \cdot 10^{11}$ \\
$\theta_{5}$ & $2.10 \cdot 10^{11}$ & $1.92 \cdot 10^{11}$ & $2.15 \cdot 10^{11}$ \\
$\theta_{6}$ & $2.02 \cdot 10^{11}$ & $2.26 \cdot 10^{11}$ & $2.17 \cdot 10^{11}$ \\
$\theta_{7}$ & $8.60 \cdot 10^{8}$ & $1.30 \cdot 10^{9}$ & $1.17 \cdot 10^{9}$ \\
$\theta_{8}$ & $7.48 \cdot 10^{11}$ & $1.57 \cdot 10^{12}$ & $1.31 \cdot 10^{12}$ \\
$\theta_{9}$ & $6.43 \cdot 10^{7}$ & $7.50 \cdot 10^{7}$ & $7.50 \cdot 10^{7}$ \\
$\theta_{10}$ & $7.70 \cdot 10^{6}$ & $7.61 \cdot 10^{6}$ & $7.65 \cdot 10^{6}$ \\
\hline
\end{tabular}


Finally, Table 7 shows the updated natural frequencies, $f_{\text {upd,j, }}$, the relative differences, $\Delta f_{\text {exp, }, j}^{u p d, j}$, and the $M A C_{\text {exp }, j}^{u p d . j}$ ratio for each considered vibration mode $j$ after the updating process considering the multi-objective approach and the three mentioned computational algorithms. As Table 7 shows the correlation between the experimental and numerical modal properties after the updating process is adequate for the three mentioned computational algorithms (all the relative differences, $\Delta f_{\text {exp }, j, j}^{u p}$, are lower than $5 \%$, and all the $M A C_{\text {exp,j }}^{u p d . j}$ ratios are greater than 0.9 ). 
Table 7. Updated, $f_{u p d, j}$, and experimental, $f_{\text {exp }, j}$, natural frequency, relative difference, $\Delta f_{\text {exp,j }}^{u p d}$, and the $M A C_{\text {exp,j }}^{u p d . j}$ ratio for each considered vibration mode $j$ after the updating process considering the multi-objective approach and the three mentioned computational algorithms.

\begin{tabular}{|c|c|c|c|c|c|c|c|c|c|c|}
\hline \multirow{2}{*}{$\begin{array}{c}\text { Mode } \\
(j)\end{array}$} & \multirow{2}{*}{$\begin{array}{l}f_{\text {exp }, j} \\
{[\mathrm{~Hz}]}\end{array}$} & \multicolumn{3}{|c|}{ MGA } & \multicolumn{3}{|c|}{ MHS } & \multicolumn{3}{|c|}{ UKF-MHS } \\
\hline & & $\begin{array}{r}f_{u p d, j} \\
{[\mathrm{~Hz}]}\end{array}$ & $\begin{array}{c}\Delta f_{\text {exp, }, j}^{u p d, j} \\
{[\%]}\end{array}$ & $\begin{array}{c}M A C_{e x p, j}^{u p d . j} \\
{[-]}\end{array}$ & $\begin{array}{c}f_{u p d, j} \\
{[\mathrm{~Hz}]}\end{array}$ & $\begin{array}{c}\Delta f_{\text {exp, }, j}^{u p d, j} \\
{[\%]}\end{array}$ & $\begin{array}{c}M A C_{e x p, j}^{u p d}, j \\
{[-]}\end{array}$ & $\begin{array}{r}f_{u p d, j} \\
{[\mathrm{~Hz}]}\end{array}$ & $\begin{array}{c}\Delta f_{e x p, j}^{u p d, j} \\
{[\%]}\end{array}$ & $\begin{array}{c}M A C_{e x p, j}^{u p d . j} \\
{[-]}\end{array}$ \\
\hline 1 & 3.810 & 3.900 & 2.362 & 0.998 & 3.871 & 1.601 & 0.998 & 3.872 & 1.627 & 0.999 \\
\hline 2 & 5.144 & 5.283 & 2.702 & 0.994 & 5.270 & 2.449 & 0.994 & 5.252 & 2.100 & 0.994 \\
\hline 3 & 8.485 & 8.373 & -1.320 & 0.988 & 8.360 & -1.473 & 0.988 & 8.361 & -1.461 & 0.989 \\
\hline 4 & 12.366 & 12.040 & -2.636 & 0.902 & 11.933 & -3.502 & 0.902 & 11.862 & -4.076 & 0.901 \\
\hline 5 & 18.605 & 18.360 & -1.317 & 0.985 & 18.630 & 0.134 & 0.987 & 18.479 & -0.677 & 0.987 \\
\hline 6 & 20.459 & 20.471 & 0.059 & 0.993 & 20.100 & -1.755 & 0.993 & 20.003 & -2.229 & 0.993 \\
\hline 7 & 22.980 & 21.880 & -4.787 & 0.951 & 22.420 & -2.437 & 0.951 & 22.234 & -3.246 & 0.948 \\
\hline
\end{tabular}

Finally, for the sake of completeness, Figure 8 illustrates the first seven numerical vibration modes, $\phi_{u p d, j}$, obtained after the model updating of the footbridge performed considering the multi-objective approach and the UKF-MHS algorithm. The first seven experimental vibration modes, $\phi_{\exp , j}$, have also been shown in Figure 8 (being the $j$ considered vibration mode).

Thus, it has been validated that the hybrid UKF-MHS algorithm is the most efficient algorithm among the three considered computational ones to perform the finite-element-model updating of civil engineering structure under the multi-objective approach. Additionally it has been checked that the performance of the proposed algorithm depends clearly on the considered value of the three mentioned hyperparameters $\left(N_{U K F}, \boldsymbol{P}_{0}^{\boldsymbol{\theta}}\right.$ and $\left.\mathbf{R}_{i i}\right)$. 


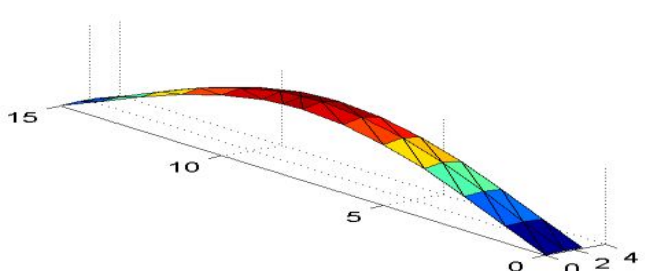

$f_{\text {upd }, 1}=3.872 \mathrm{~Hz}$

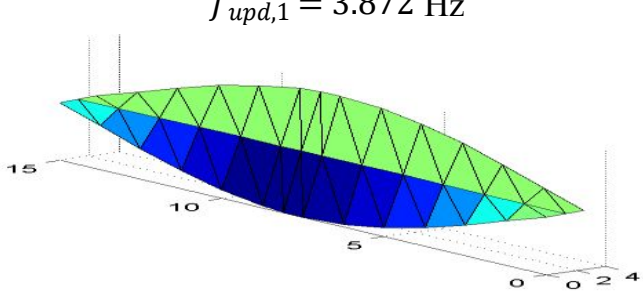

$f_{\text {upd }, 2}=5.252 \mathrm{~Hz}$

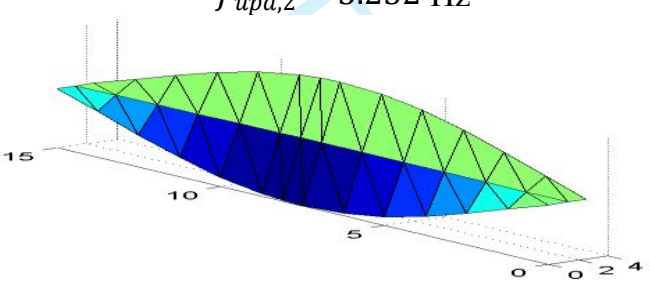

$f_{\text {upd,3 }}=8.361 \mathrm{~Hz}$

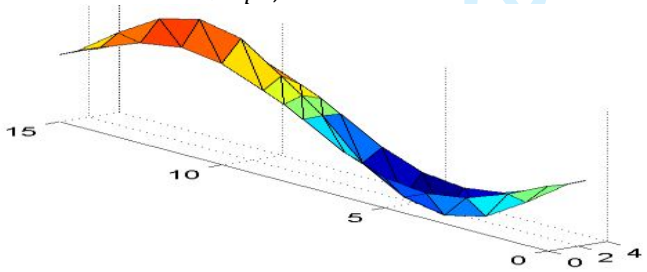

$f_{u p d, 4}=11.862 \mathrm{~Hz}$

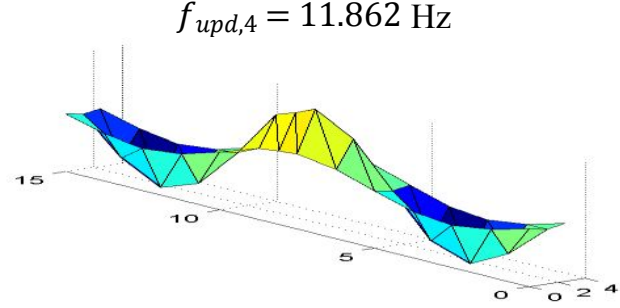

$f_{\text {upd }, 5}=18.479 \mathrm{~Hz}$

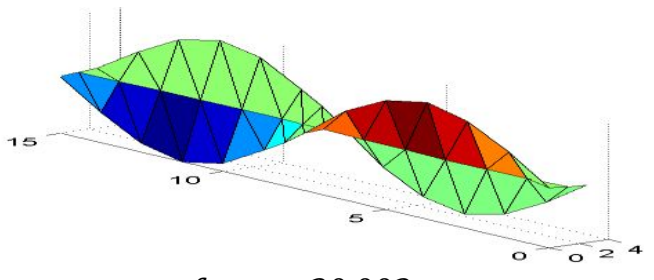

$f_{\text {upd,6 }}=20.003 \mathrm{~Hz}$

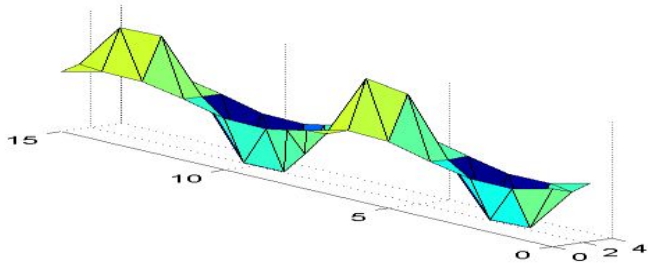

$f_{u p d, 7}=22.234 \mathrm{~Hz}$

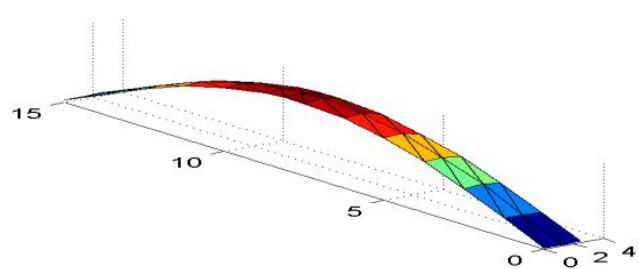

$f_{\text {exp }, 1}=3.810 \mathrm{~Hz}$

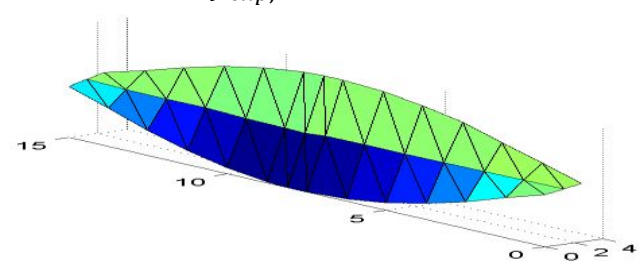

$f_{\text {exp }, 2}=5.144 \mathrm{~Hz}$

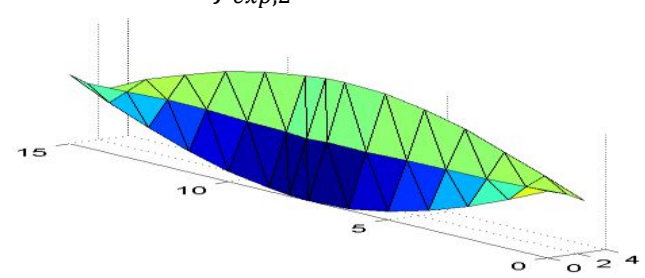

$f_{\exp , 3}=8.485 \mathrm{~Hz}$

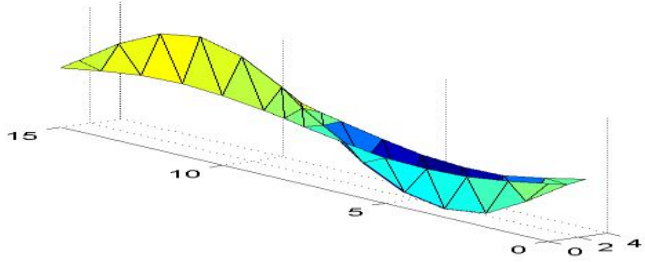

$f_{\text {exp }, 4}=12.366 \mathrm{~Hz}$

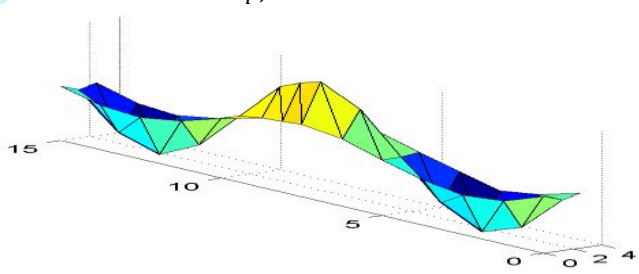

$f_{\text {exp }, 5}=18.605 \mathrm{~Hz}$

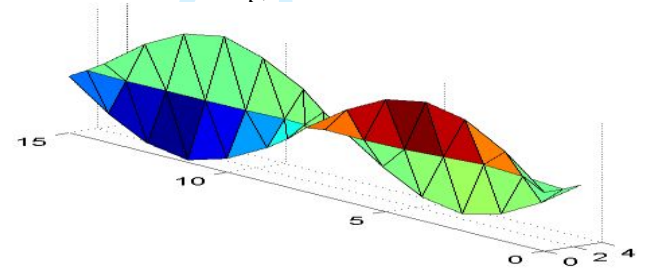

$f_{\exp , 6}=20.459 \mathrm{~Hz}$

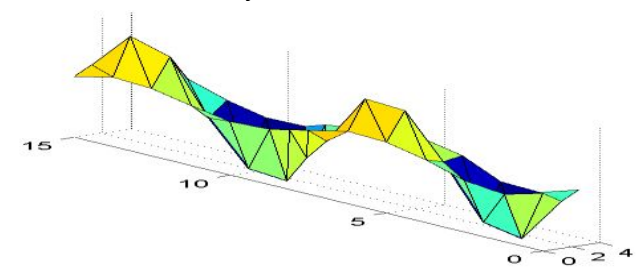

$f_{\text {exp }, 7}=22.980 \mathrm{~Hz}$

Figure 8. First seven updated, $\phi_{u p d, j}$, and experimental, $\phi_{\text {exp }, j}$, vibration modes of the laboratory footbridge (being $j$ the considered vibration mode). 


\section{Conclusions}

FE model updating of civil engineering structures is usually performed under the maximum likelihood method. According to this method, the FE model updating problem is transformed into an optimization problem. Due to the complexity and the non-linear behaviour of the resulting objective function, computational intelligence algorithms are usually employed to solve this optimization problem. Among these computational algorithms, nature-inspired computational algorithms are especially effective to tackle this problem. Nevertheless, the use of these algorithms has a clear drawback, the high simulation time required to perform the updating process. In order to overcome this limitation, a hybrid UFK-HS optimization algorithm has been proposed, implemented and validated herein.

The proposed algorithm consists in combining two individual algorithms: (i) a local optimization algorithm, the square root unscented Kalman filter; and (ii) a global optimization algorithm, the harmony search algorithm.

The FE model updating of a real structure, a laboratory footbridge located at the University of Exeter (U.K.), has been considered herein to validate the performance of the new hybrid algorithm. The experimental modal properties of the structure were obtained by the signal processing (experimental modal analysis) of the records obtained during a forced vibration test. For comparison purposes, the FE model updating has been performed under two approaches (single-objective and multi-objective approaches) and considering three different computational intelligence algorithms: (i) genetic algorithms; (ii) harmony search; and (iii) the proposed hybrid UKF-HS algorithm.

Two comparison criteria have been considered herein: (i) the convergence speed; and (ii) the accuracy of the adjustment. The proposed hybrid UKF-HS algorithm has been shown as the most efficient algorithms to perform the finite-element-model updating of the laboratory footbridge under the two approaches (single-objective and multi-objective). Additionally, a sensitivity study has been performed to analysis the performance of the hybrid algorithm under the variation of three hyper-parameters: (i) the number of iterations of the UKF algorithm, $N_{U F K}$; (ii) the initial estimation error covariance, $\boldsymbol{P}_{0}^{\boldsymbol{\theta}}$; and (iii) the measurement noise covariance matrix, $\mathbf{R}_{i i}$. As result of this study, an adequate value of these hyper-parameters must be considered to optimize the performance of the proposed hybrid algorithm.

Therefore, the proposed hybrid UKF-HS algorithm is an available tool to perform the finite-element-model updating of civil engineering structures for practical engineering applications. Nevertheless, further studies are needed to validate the performance of the proposed hybrid algorithm for the finite-element-model updating of different types of civil engineering structures.

\section{Acknowledgements}

This work was partially funded by the Ministerio de Economía y Competitividad of Spain and the European Regional Development Fund under project RTI2018094945-B-C21. Additionally, the co-author, J. Naranjo-Pérez, has been supported by the research contract, USE-17047-G, provided by the Universidad de Sevilla. 


\section{References}

Allemang RJ, Brown DL (1982). A correlation coefficient for modal vector analysis. Proc. $1^{\text {st }}$ Int. Modal Anal. Conf., vol. 1, Orlando: Union College Press, p. 110116.

Ansys Mechanical 19.0. Ansys Inc 2019 n.d. http://www.ansys.com (accessed May 2019).

Astroza R, Barrientos N, Li Y, Saavedra Flores E (2019). Calibration of a large nonlinear finite element model of a highway bridge with many uncertain parameters. Proc. IMAC XXXVII, Orlando (USA).

Astroza R, Nguyen LT, Nestorović T (2016). Finite element model updating using simulated annealing hybridized with unscented Kalman filter. Computer and Structures, 177: 176-191. doi: https://doi.org/10.1016/j.compstruc.2016.09.001.

Bartilson DT, Jang J, Smyth AW (2019). Finite element model updating using objective-consistent sensitivity-based parameter clustering and Bayesian regularization. Mechanical Systems and Signal Processing, 114: 328-345. doi: https://doi.org/10.1016/j.ymssp.2018.05.024.

Beck JL, Au S-K (2002). Bayesian updating of structural models and reliability using Markov chain Monte Carlo simulation. Journal of Engineering Mechanics, 128: 380-391. doi: https://doi.org/10.1061/(ASCE)0733-9399(2002)128:4(380).

Beck JL, Au S-K, Vanik MW (2001). Monitoring structural health using a probabilistic measure. Computer-Aided Civil and Infrastructure Engineering, 16: 1-11. doi: http://dx.doi.org/10.1111/0885-9507.00209.

Beck JL, Katafygiotis LS (1998). Updating models and their uncertainties. I: Bayesian statistical framework. Journal of Engineering Mechanics, 124: 455-461. doi: http://dx.doi.org/10.1061/(ASCE)0733-9399(1998)124:4(455).

Cheung SH, Beck JL (2009). Bayesian model updating using hybrid Monte Carlo simulation with application to structural dynamic models with many uncertain parameters. Journal of Engineering Mechanics, 135: 243-255. doi: https://doi.org/10.1061/(ASCE)0733-9399(2009)135:4(243).

Ching J, Chen Y-C (2007). Transitional Markov chain Monte Carlo method for Bayesian model updating, model class selection, and model averaging. Journal of Engineering Mechanics, 133: 816-832. doi: https://doi.org/10.1061/(ASCE)0733-9399(2007)133:7(816). 
Ching J, Muto M, Beck JL (2006). Structural model updating and health monitoring with incomplete modal data using Gibbs sampler. Computer-Aided Civil Infrastructure Engineering, 21: 242-257. doi: https://doi.org/10.1111/j.1467$\underline{8667.2006 .00432 . x}$.

Clarke, JL (1996). Structural Design of Polymer Composites - EUROCOMP Design Code and Handbook. E\&FN Spon.

Deb K, Gupta S (2011). Understanding knee points in bicriteria problems and their implications as preferred solution principles. Engineering Optimization 43: 1175-1204. doi: https://doi.org/10.1080/0305215X.2010.548863.

Deb K, Pratap A, Agarwal S, Meyarivan T (2002). A fast and elitist multi-objective genetic algorithm: NSGA-II. IEEE Transactions on Evolutionary Computation, 6: 182-197. doi: https://doi.org/10.1109/4235.996017.

Eurocode 3 (2005): Design of steel structures. CEN.

Fadel L, Fadel L, Kaminski Jr J, Riera JD (2012). Damage detection under ambient vibration by harmony search algorithm. Expert Systems with Applications, 39(10): 9704-9714. doi: https://doi.org/10.1016/j.eswa.2012.02.147.

Fan W, Qiao P (2011). Vibration-based damage identification methods: a review and comparative study. Structural Health Monitoring, 10(1):83-111. doi: http://dx.doi.org/10.1177/1475921710365419.

Fox R, Kapoor M (1968). Rates of change of eigenvalues and eigenvectors. AIAA Journal, 6: 2426-2429. doi: https://doi.org/10.2514/3.5008.

Friswell M, Mottershead JE (1995). Finite element model updating in structural dynamics. vol. 38. Springer Science \& Business Media.

Fritzen C-P, Jennewein D, Kiefer T (1998). Damage detection based on model updating methods. Mechanical System and Signal Processing, 12: 163-186. doi: http://dx.doi.org/10.1006/mssp.1997.0139.

Geem ZW, Kim JH, Loganathan GV (2001). A new heuristic optimization algorithm: harmony search. Simulation, 76:60-68. doi: https://doi.org/10.1177/003754970107600201.

Guo J, Zhao X, Guo J, Yuan X, Dong S, Xiong Z (2017). Model updating of suspendeddome using artificial neural networks. Advances in Structural Engineering, 20: 1727-1743. doi: https://doi.org/10.1177/1369433217693629. 
Hao H, Xia Y (2002). Vibration-based damage detection of structures by genetic algorithm. Journal of Computing in Civil Engineering, 16: 222-229. doi: https://doi.org/10.1061/(ASCE)0887-3801(2002)16:3(222).

Hasançebi O, Dumlupınar T (2013). Linear and nonlinear model updating of reinforced concrete T-beam bridges using artificial neural networks. Computer and Structures, 119: 1-11. doi: https://doi.org/10.1016/j.compstruc.2012.12.017.

Hudson EJ, Reynolds P (2017). Design and Construction of a Reconfigurable Pedestrian Structure. Experimental Techniques. doi: http://dx.doi.org/10.1007/s40799-016$\underline{0144-3}$.

Infantes M, Naranjo-Pérez J, Jiménez-Alonso JF, Sáez A (2019). Determining the Best Pareto-solution in a Multi-Objective Approach for Model Updating, IABSE Symposium, Guimaraes 2019: Towards a Resilient Built Environment Risk and Asset Management - Report pp. 523-530

Jazwinski, AH (1970). Stochastic processes and filtering theory, Academic Press.

Jiménez-Alonso JF, Hudson EJ, Pavic A, Sáez A (2017) Maximum Likelihood Methods for Finite Element Model Updating of Civil Engineering Structures: A Comparative Study. In Proceedings of the $4^{\text {th }}$ International Conference on Mechanical Models in Structural Engineering CMMoST 2017, Madrid (Spain)

Jiménez-Alonso JF, Hudson EJ, Pavic A, Sáez A (2019). Probabilistic Finite Element Model Updating of Civil Engineering Structures: A Comparative Study, IABSE Symposium, Guimaraes 2019: Towards a Resilient Built Environment Risk and Asset Management - Report pp. 1269-1276

Jiménez-Alonso JF, Sáez A (2016). Model Updating for the Selection of an Ancient Bridge Retrofitting Method in Almeria, Spain. Structural Engineering International, 26: 17-26. doi: https://doi.org/10.2749/101686615X14355644771333.

Jin S, Cho S, Jung H, Lee J and Yun C (2014) A new multi-objective approach to finite element model updating. Journal of Sound and Vibration. 333(11): 2323-2338. doi: https://doi.org/10.1016/i.jsv.2014.01.015.

Julier SJ, Uhlmann JK (1997). New extension of the Kalman filter to nonlinear systems. Proceedings of the SPIE 3068, Signal Processing, Sensor Fusion, and Target Recognition VI 182-194. doi: https://doi.org/10.1117/12.280797. 
Julier SJ, Uhlmann JK (2004). Corrections to "Unscented filtering and nonlinear estimation". Proceedings of the IEEE 2004, 92: 1958. doi: https://doi.org/10.1109/JPROC.2004.837637.

Kalman RE (1960). A new approach to linear filtering and prediction problems. Journal of Basic Engineering, 82: 35-45. doi: https://doi.org/10.1115/1.3662552.

Kaveh A, Javadi SM, Maniat M (2014). Damage assessment via modal data with a mixed particle swarm strategy, ray optimizer, and harmony search. Asian Journal of Civil Engineering, 15(1): 95-106.

Koh CG, Perry MJ (2009). Structural Identification and Damage Detection using Genetic Algorithms: Structures and Infrastructures Book Series, Vol. 6. Crc Press.

Levin RI, Lieven NAJ (1998a). Dynamic finite element model updating using neural networks. Journal of Sound and Vibration, 210: 593-607. doi: https://doi.org/10.1006/jsvi.1997.1364.

Levin RI, Lieven NAJ (1998b). Dynamic finite element model updating using simulated annealing and genetic algorithms. Mechanical System and Signal Processing, 12: 91-120. doi: https://doi.org/10.1006/mssp.1996.0136.

Lu Y, Tu Z (2004). A two-level neural network approach for dynamic FE model updating including damping. Journal of Sound and Vibration, 275: 931-952. doi: https://doi.org/10.1016/S0022-460X(03)00796-X.

Maia NMM, e Silva JMM. Theoretical and experimental modal analysis. Research Studies Press; 1997.

Manjarres D, Landa-Torres I, Gil-Lopez S, Del Ser J, Bilbao MN, Salcedo-Sanz S, et al (2013). A survey on applications of the harmony search algorithm. Engineering Applications of Artificial Intelligence, 26: 1818-1831. doi; https://doi.org/10.1016/j.engappai.2013.05.008.

Marwala T. (2010). Finite-Element-Model Updating Using Computational Intelligence Techniques. Applications to Structural Dynamics, p. 231. Springer, London.

Marwala T, Boulkaibet I, Adhikari S (2016). Probabilistic finite element model updating using bayesian statistics: applications to aeronautical and mechanical engineering. John Wiley \& Sons.

Matlab Inc. MATLAB R2019a n.d. http://www.mathworks.com (accessed May 2019) 
Mottershead JE, Link M, Friswell MI (2011). The sensitivity method in finite element model updating: a tutorial. Mechanical Systems and Signal Processing, 25: 2275-2296. doi: https://doi.org/10.1016/j.ymssp.2010.10.012.

Nguyen LT, Nestorović T (2015). Nonlinear Kalman filters for model calibration of soil parameters for geomechanical modeling in mechanized tunneling. Journal of Computing in Civil Engineering, 30: 04015025. doi: https://doi.org/10.1061/(ASCE)CP.1943-5487.0000495.

Nocedal J, Wright SJ (1999) Numerical optimization. Springer Series in Operations Research.

Papadimitriou C, Argyris C, Panetsos, P (2018). Information-driven modeling of structures using Bayesian framework. In: Conte J, Astroza R, Benzoni G, Feltrin G, Loh K, Moaveni B. (eds) Experimental Vibration Analysis for Civil Structures. EVACES 2017. Lecture Notes in Civil Engineering, vol 5. Springer, Cham.

Perera R, Fang S-E, Ruiz A (2010). Application of particle swarm optimization and genetic algorithms to multi-objective damage identification inverse problems with modelling errors. Meccanica, 45: 723-734. doi: https://doi.org/10.1007/s11012-009-9264-5.

Robert CP, Casella G (1999). The Metropolis-Hastings Algorithm. In: Monte Carlo Statistical Methods. Texts in Statistics. Springer, New York, NY pp. 231-283. doi: https://doi.org/10.1007/978-1-4757-3071-5 6.

Shabbir F, Omenzetter P (2015). Particle swarm optimization with sequential niche technique for dynamic finite element model updating. Computer-Aided Civil and Infrastructure Engineering, 30: 359-375. doi: https://doi.org/10.1111/mice.12100.

SPS (2019). Sandwich Plate System heavy engineering composite from Intelligent Engineering n.d. http:/www.ie-sps.com/ (accessed May 2019).

Tarantola A (2005). Inverse problem theory and methods for model parameter estimation. vol. 89. SIAM; doi: https://doi.org/10.1137/1.9780898717921.

Terejanu GA (2011). Unscented Kalman filter tutorial. Univ Buffalo Buffalo.

Teughels A, Maeck J, De Roeck G (2002). Damage assessment by FE model updating using damage functions. Computer and Structures, 80: 1869-1879. doi: http://dx.doi.org/10.1016/50045-7949(02)00217-1. 
Vakilzadeh MK, Yaghoubi V, Johansson AT, Abrahamsson T. (2014). Manifold Metropolis adjusted Langevin algorithm for high-dimensional Bayesian FE model updating in structural dynamics. Proceedings of the $9^{\text {th }}$ International Conference on Structural Dynamics, EURODYN 2014, Porto (Portugal).

Van Der Merwe R (2004). Sigma-point Kalman filters for probabilistic inference in dynamic state-space models. PhD Thesis. Oregon Health \& Science University.

Van Der Merwe R, Wan EA (2001). The square-root unscented Kalman filter for state and parameter-estimation. Proceedings of the International Conference on Acoustics, Speech, and Signal Processing. Proceedings, 6, 3461-3464. doi: http://dx.doi.org/10.1109/ICASSP.2001.940586.

Wan EA, Van Der Merwe R (2000). The unscented Kalman filter for nonlinear estimation. Proceedings of the IEEE 2000 Adaptive Systems for Signal Processing, Communications, and Control Symposium; 153-158. doi: http://dx.doi.org/10.1109/ASSPCC.2000.882463.

Wan EA, Van Der Merwe R, Nelson AT (2000). NIPS'99 Proceedings of the 12th International Conference on Neural Information Processing Systems, 666-672.

Wang Y, Li ZX, Wang CM (2011). Concurrent multifactor optimisation techniques for model updating of long-span bridges. Structure and Infrastructure Engineering, 9(6): 578-593. doi: https://doi.org/10.1080/15732479.2011.595801.

Wang Y-O, Zong L, Shi Y-J, Yao N (2014). Damage detection and rehabilitation on a curvilinear steel box girder bridge by multistage model updating. Structure and Infrastructure Engineering, 11(11): 1420-1431. doi: https://doi.org/10.1080/15732479.2014.970202.

Yang X-S, Koziel S (2011). Computational optimization and applications in engineering and industry. vol. 359. Springer Science \& Business Media.

Živanović S, Pavic A, Reynolds, P (2007). Finite element modelling and updating of a lively footbridge: The complete process. Journal of Sound and Vibration, 301 (1-2): 126-145 doi: https://doi.org/10.1016/j.jsv.2006.09.024. 


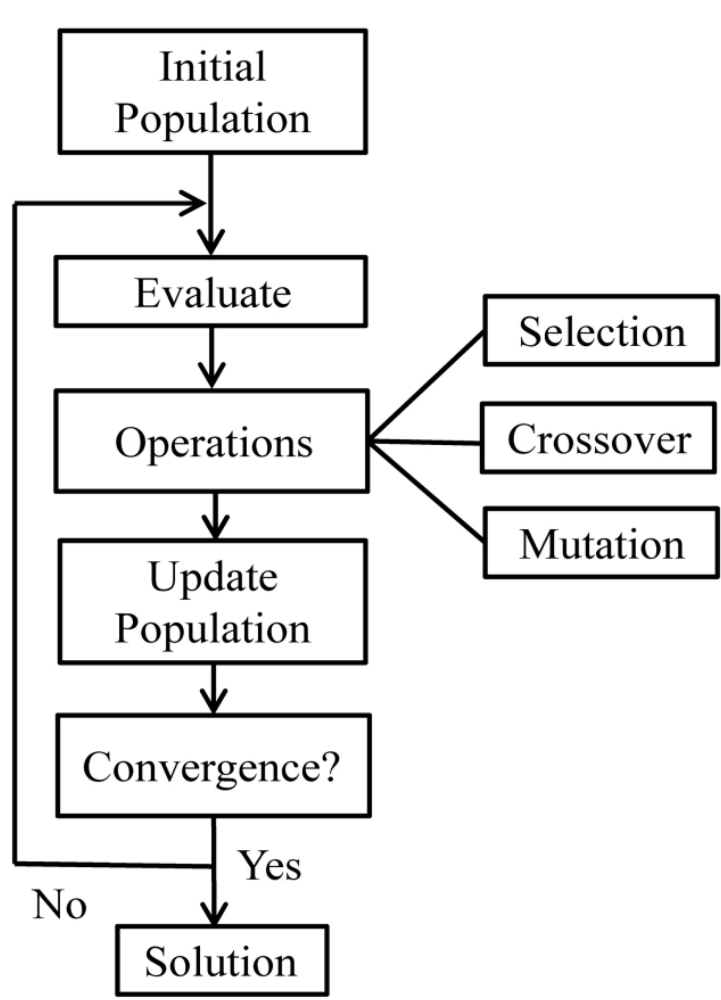

a)

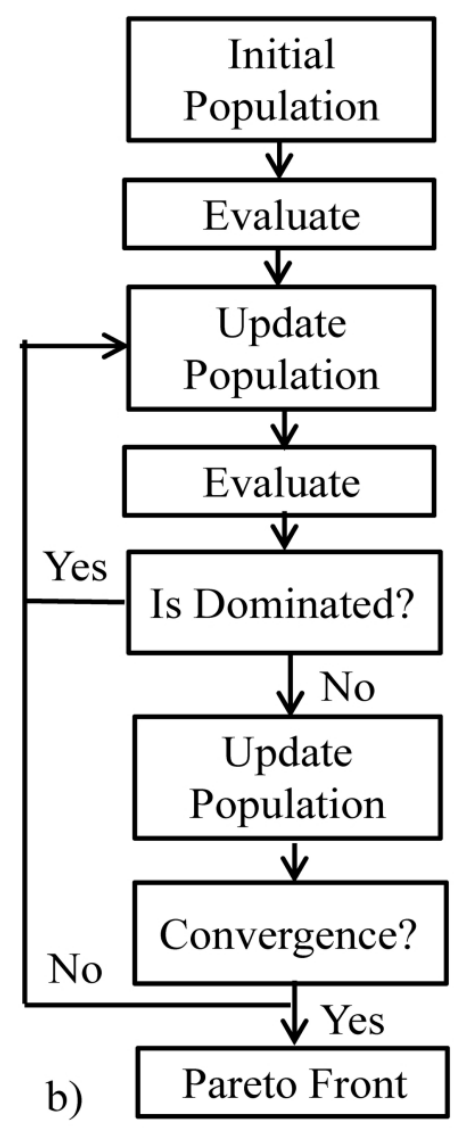

Figure 1

$125 \times 109 \mathrm{~mm}(600 \times 600 \mathrm{DPI})$ 


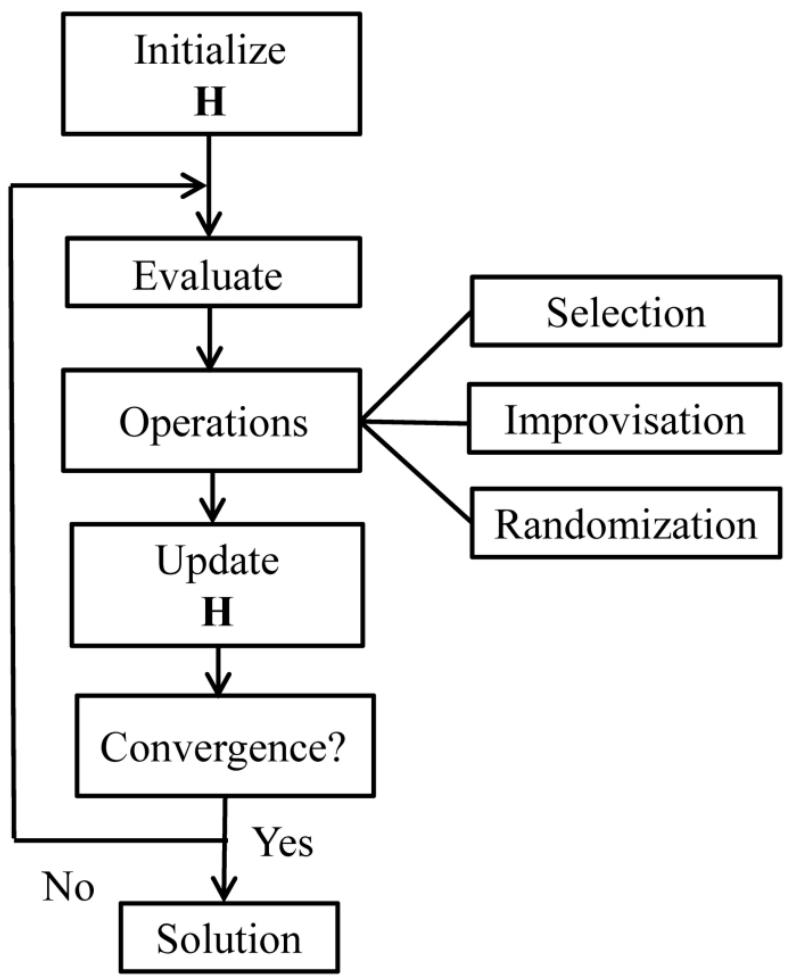

a)

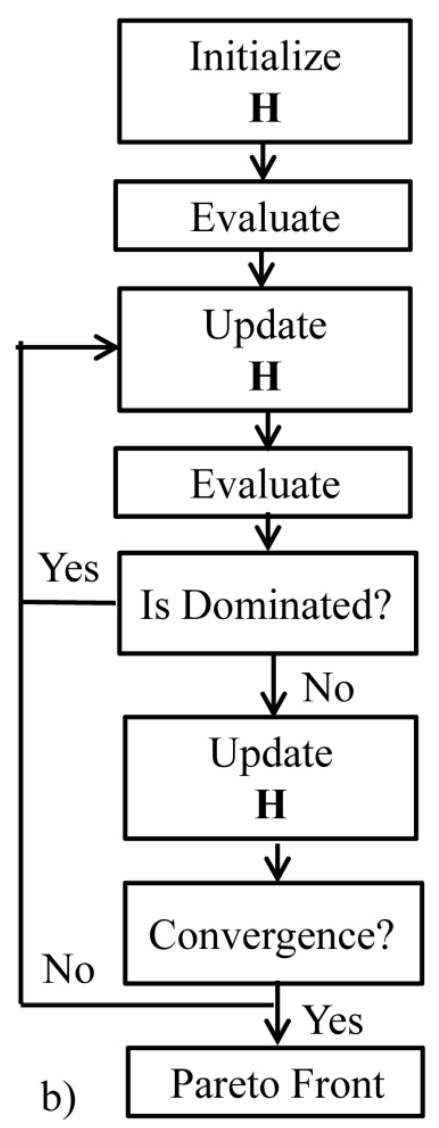

Figure 2

$126 \times 111 \mathrm{~mm}(600 \times 600 \mathrm{DPI})$ 


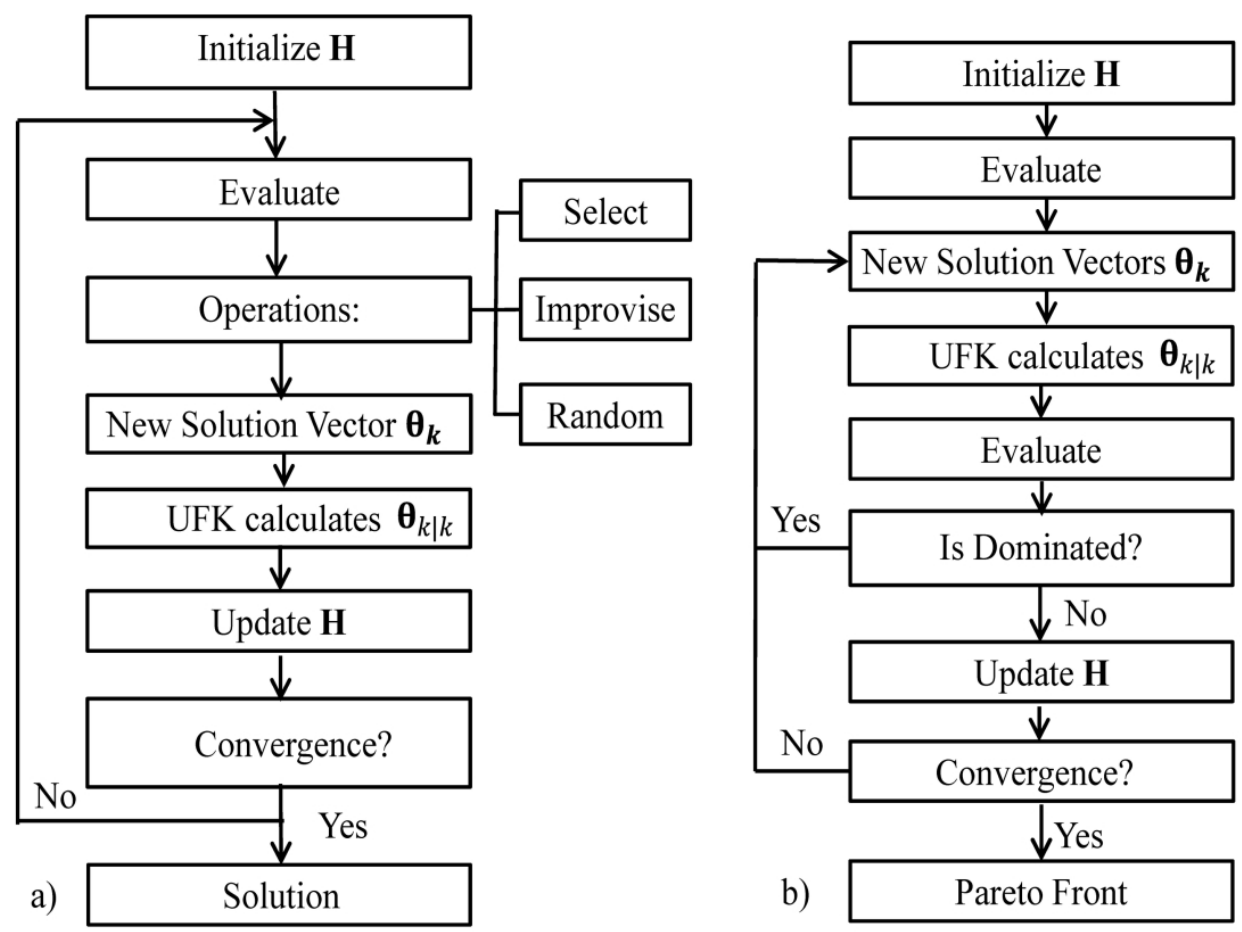

Figure 3

$126 \times 92 \mathrm{~mm}(600 \times 600 \mathrm{DPI})$ 


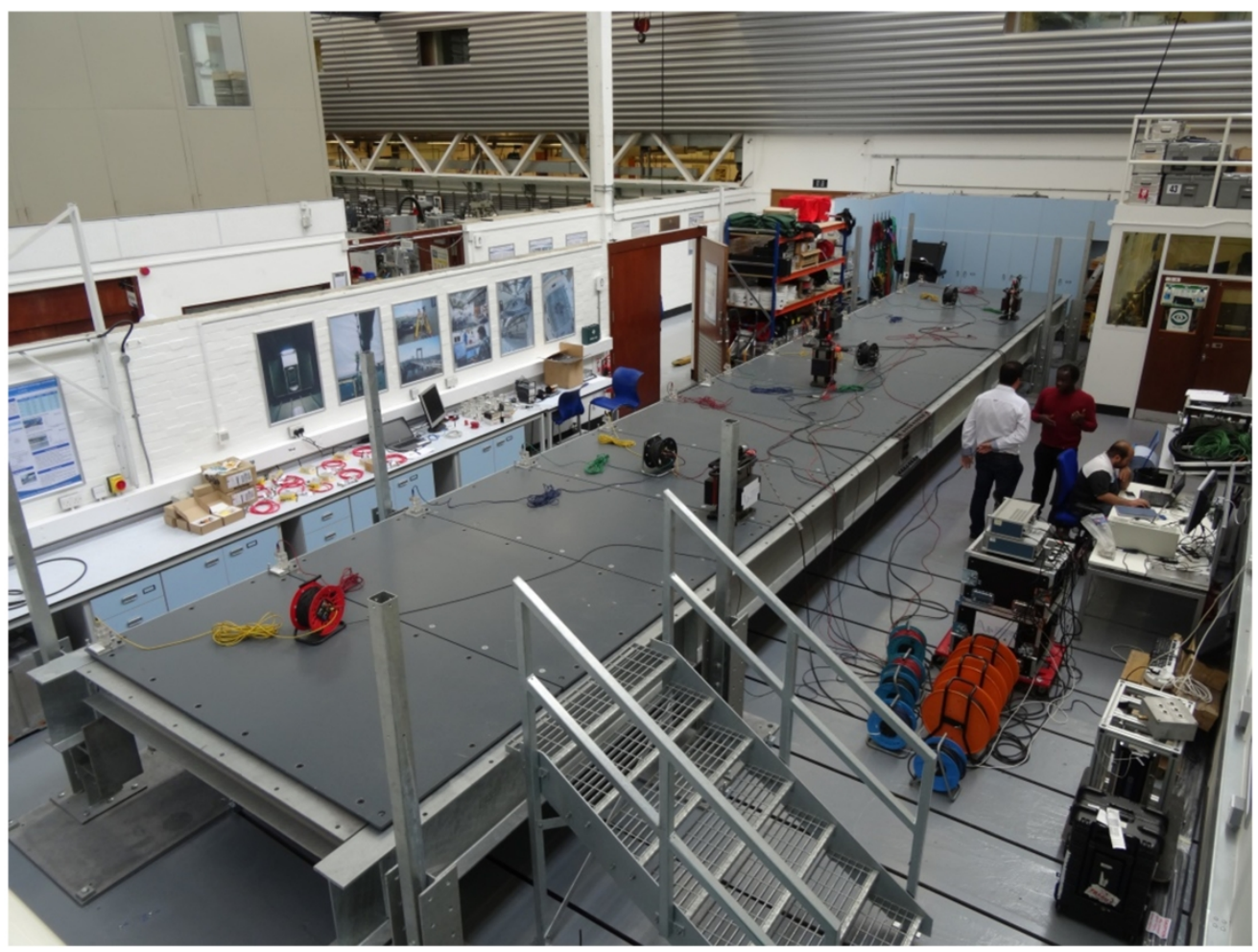

Figure 4

$130 \times 98 \mathrm{~mm}(600 \times 600 \mathrm{DPI})$ 


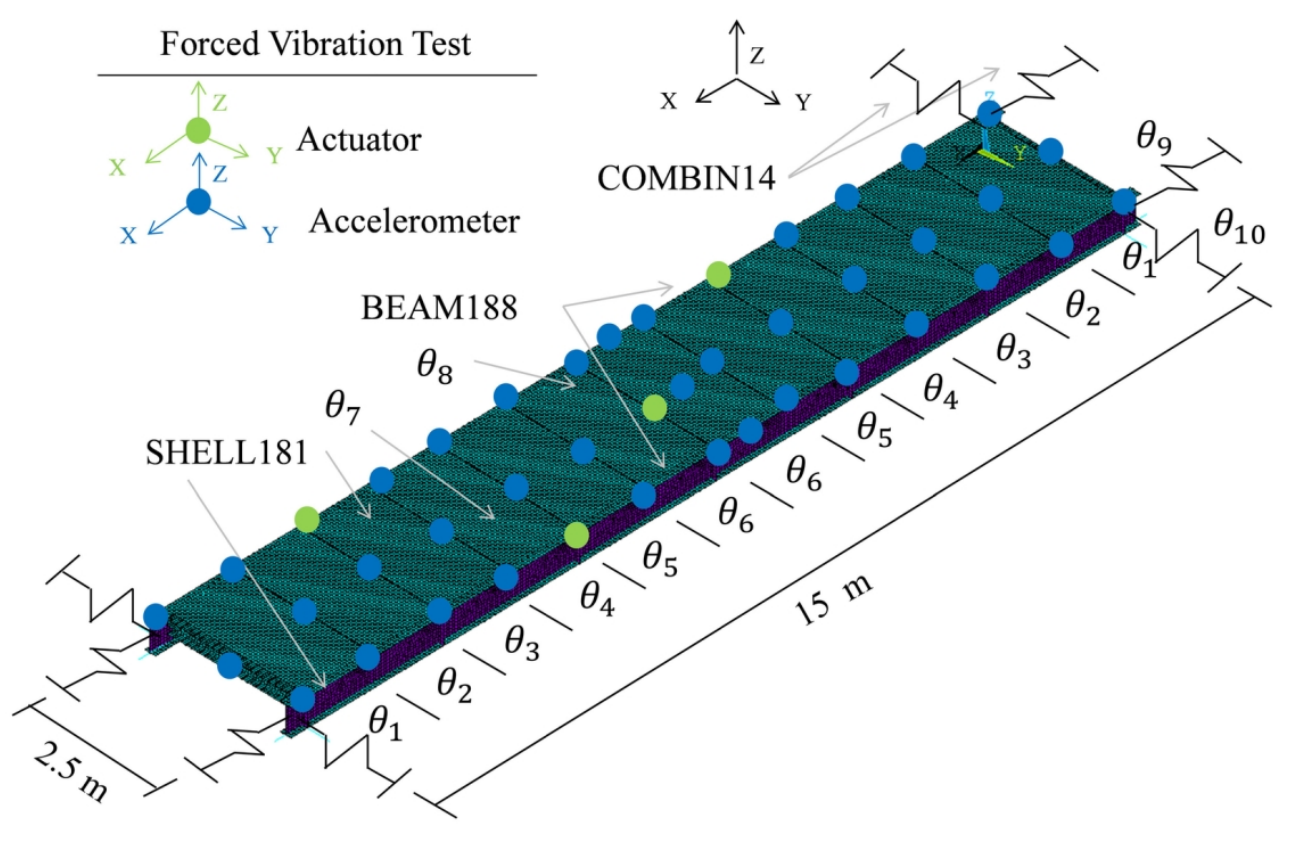

Figure 5

$140 \times 90 \mathrm{~mm}(300 \times 300$ DPI) 

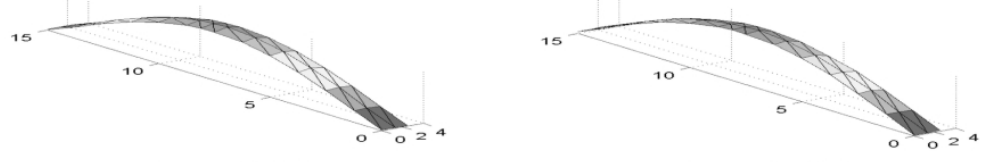

$f_{\text {num }, 1}=3.652 \mathrm{~Hz}$

$f_{\text {exp, } 1}=3.810 \mathrm{~Hz}$
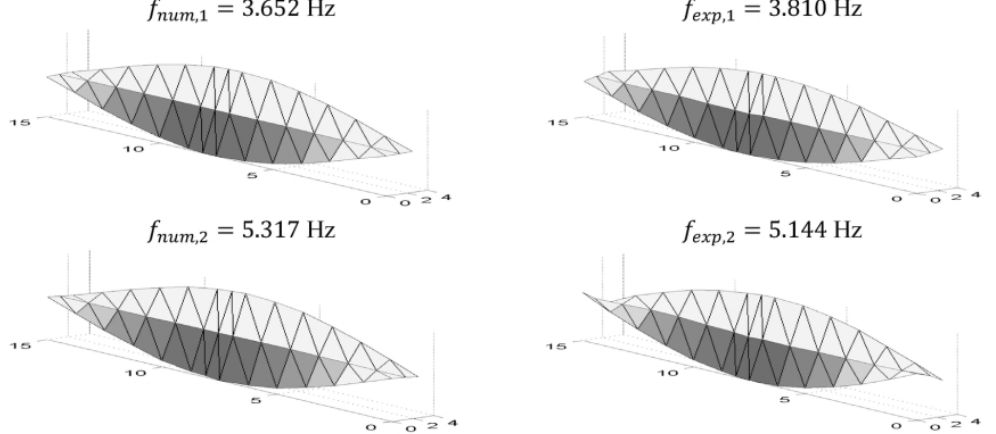

$f_{\text {exp }, 2}=5.144 \mathrm{~Hz}$

$f_{\text {num }, 3}=8.990 \mathrm{~Hz}$
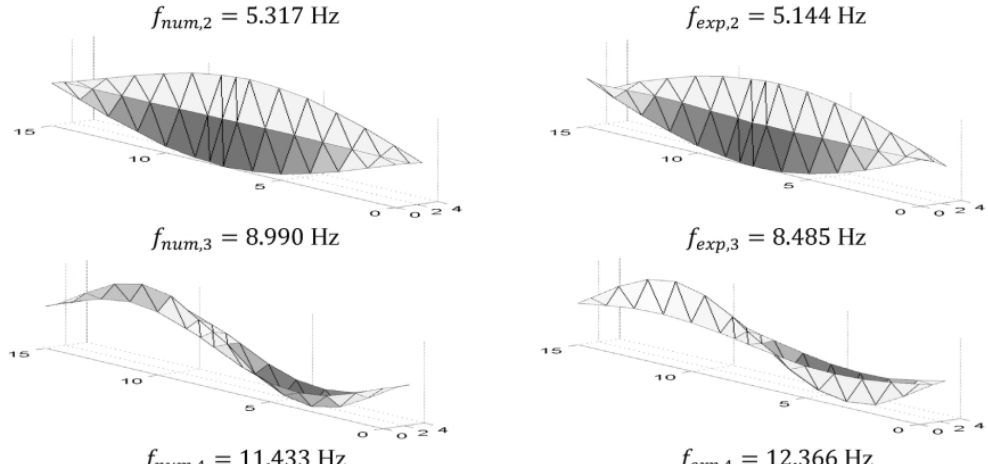

$f_{\text {exp }, 3}=8.485 \mathrm{~Hz}$
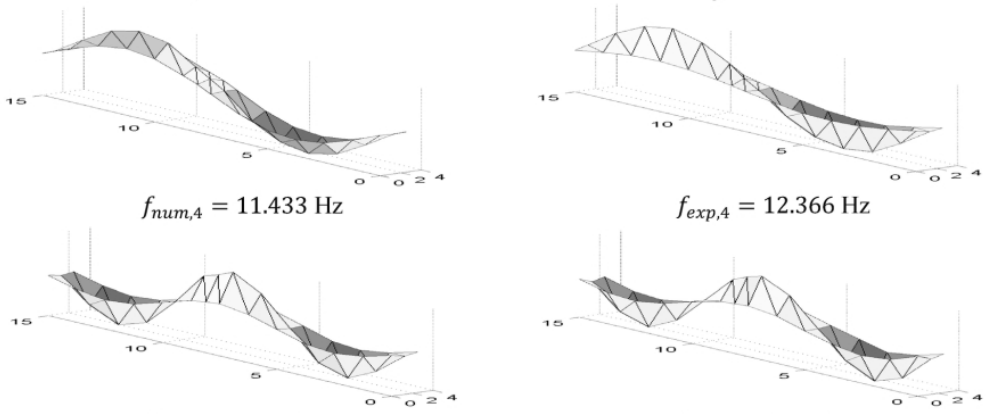

$f_{\exp , 4}=12.366 \mathrm{~Hz}$

$f_{\text {num }, 5}=17.795 \mathrm{~Hz}$
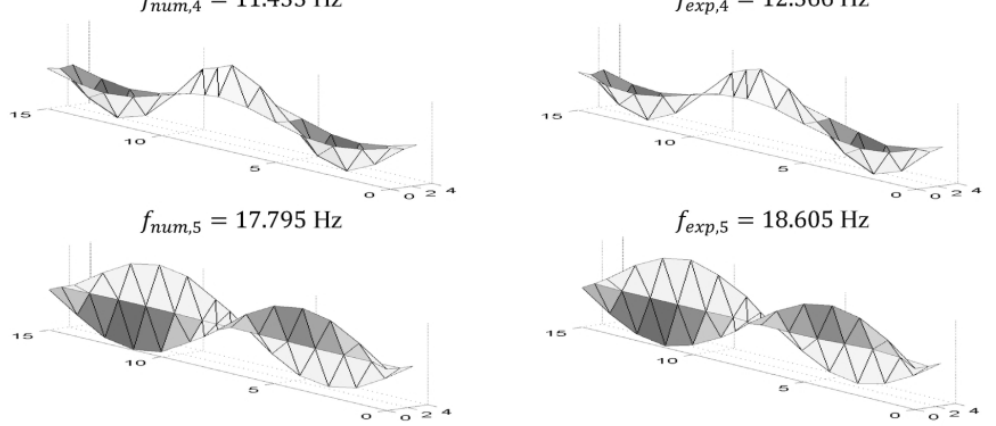

$f_{\text {exp }, 5}=18.605 \mathrm{~Hz}$

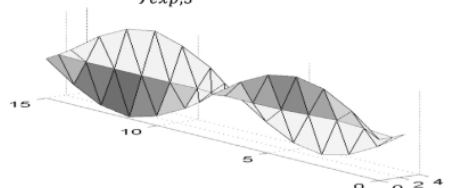

$f_{\text {num }, 6}=19.519 \mathrm{~Hz}$

$f_{\text {exp }, 6}=20.459 \mathrm{~Hz}$

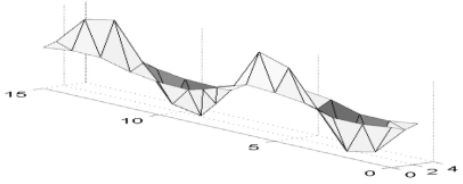

$f_{\text {num }, 7}=20.725 \mathrm{~Hz}$

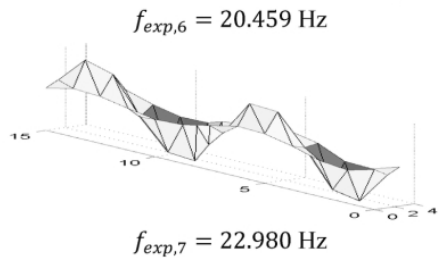

Figure 6

$148 \times 226 \mathrm{~mm}(300 \times 300$ DPI $)$

URL: http:/mc.manuscriptcentral.com/nsie 


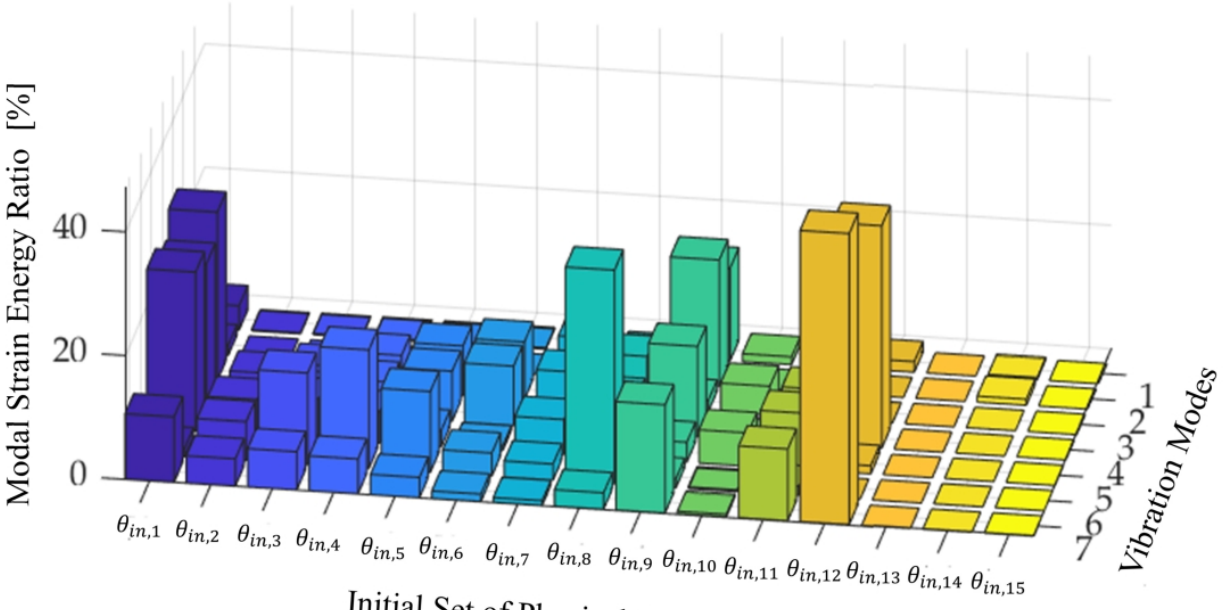

Initial Set of Physical Parameters

Figure 7

$144 \times 75 \mathrm{~mm}(600 \times 600$ DPI $)$ 

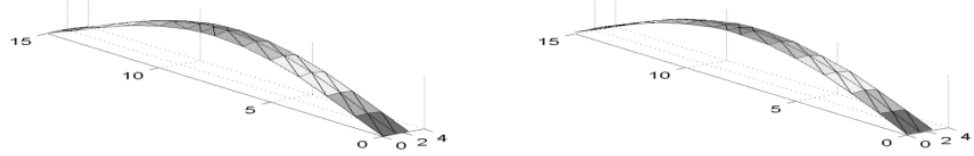

$f_{\text {upd }, 1}=3.872 \mathrm{~Hz}$

$f_{\text {exp }, 1}=3.810 \mathrm{~Hz}$
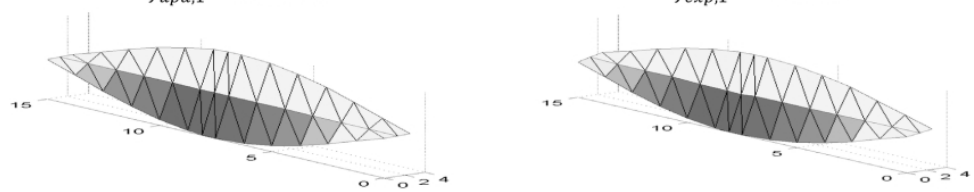

$f_{\text {upd }, 2}=5.252 \mathrm{~Hz}$

$f_{\exp , 2}=5.144 \mathrm{~Hz}$
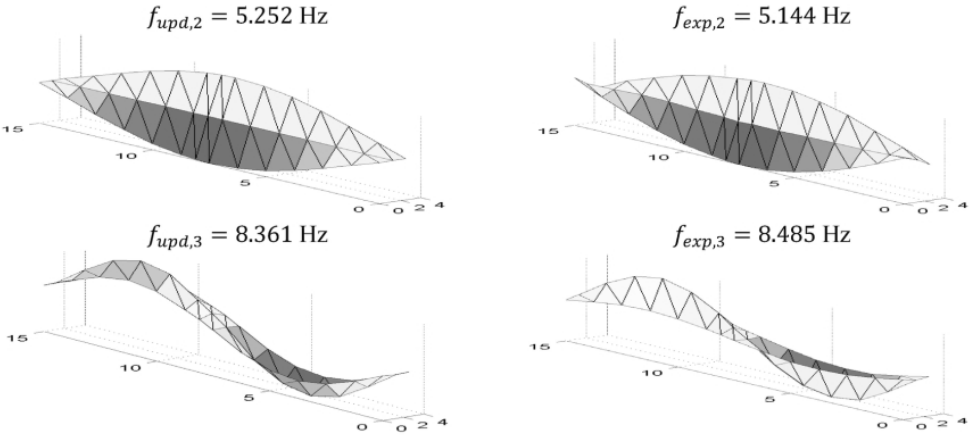

$f_{\text {exp }, 3}=8.485 \mathrm{~Hz}$

$f_{\text {upd }, 4}=11.862 \mathrm{~Hz}$
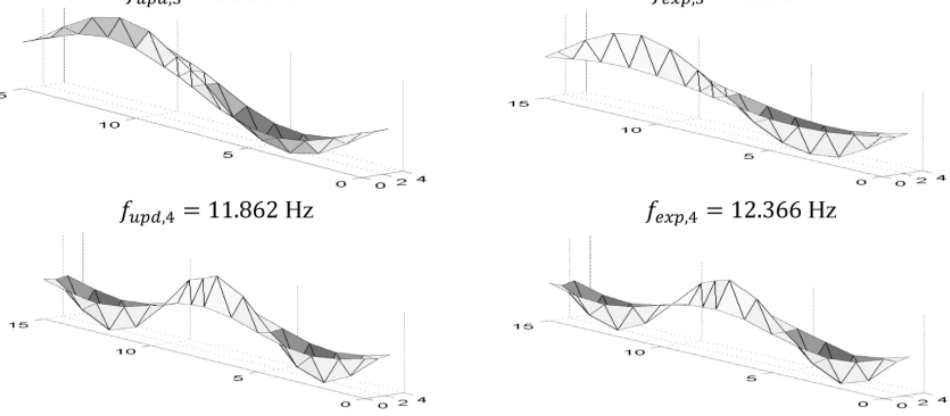

$f_{\text {exp }, 4}=12.366 \mathrm{~Hz}$

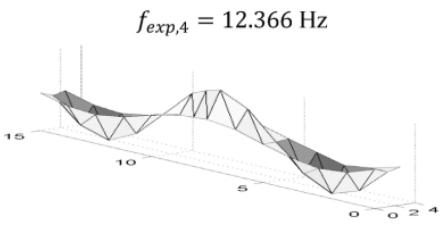

$f_{\text {upd }, 5}=18.479 \mathrm{~Hz}$

$f_{\text {exp }, 5}=18.605 \mathrm{~Hz}$
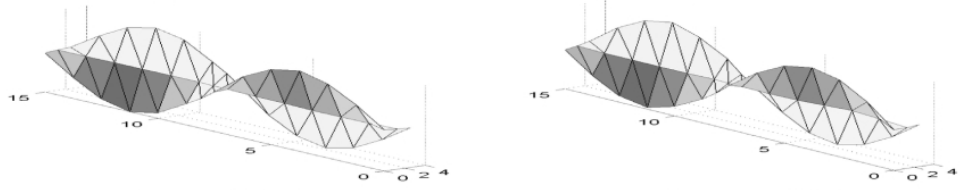

$f_{\text {upd }, 6}=20.003 \mathrm{~Hz}$

$f_{\text {exp }, 6}=20.459 \mathrm{~Hz}$

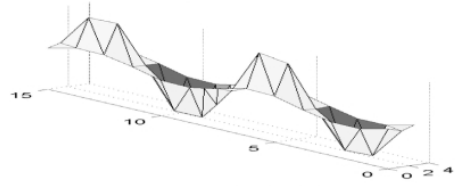

$f_{\text {upd }, 7}=22.234 \mathrm{~Hz}$

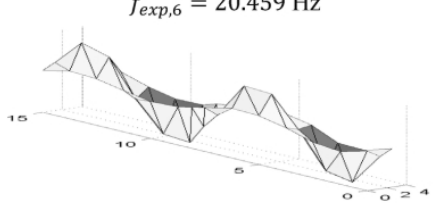

$f_{\text {exp }, 7}=22.980 \mathrm{~Hz}$

Figure 8

$149 \times 229 \mathrm{~mm}(300 \times 300 \mathrm{DPI})$ 
Table 1. Numerical, $f_{\text {num }, j}$, and experimental, $f_{\text {exp }, j}$, natural frequency, relative difference, $\Delta f_{\text {exp }, j}^{n u m}$, and the $M A C_{\text {exp }, j}^{n u m}, j$ ratio of the considered vibration mode $j$.

\begin{tabular}{ccccc}
$\begin{array}{c}\text { Mode } \\
(j)\end{array}$ & $\begin{array}{c}f_{\text {num }, j} \\
{[\mathrm{~Hz}]}\end{array}$ & $\begin{array}{c}f_{\text {exp }, j} \\
{[\mathrm{~Hz}]}\end{array}$ & $\begin{array}{c}\Delta f_{\text {exp }, j}^{\text {num } j} \\
{[\%]}\end{array}$ & $\begin{array}{c}M A C_{\text {exp }, j}^{\text {num } j} \\
{[-]}\end{array}$ \\
\hline 1 & 3.652 & 3.810 & -4.147 & 0.998 \\
2 & 5.317 & 5.144 & 3.363 & 0.994 \\
3 & 8.990 & 8.485 & 5.952 & 0.988 \\
4 & 11.433 & 12.366 & -7.545 & 0.877 \\
5 & 17.795 & 18.605 & -4.354 & 0.986 \\
6 & 19.519 & 20.459 & -4.595 & 0.993 \\
7 & 20.725 & 22.980 & -9.813 & 0.634
\end{tabular}


Table 2. Performance of the three considered algorithms to solve the updating problem under the single-objective approach (where Iter is the number of iterations of the main algorithm; Pop is the population size; $N_{U K F}$ is the number of iterations of the UKF algorithm; $\boldsymbol{P}_{0}^{\boldsymbol{\theta}}$ is the initial estimation error covariance; $\mathbf{R}_{i i}$ is the measurement noise covariance matrix; Eval is the number of evaluations of the objective function; Time is the time [s] required until a convergence criterion is met; and $f_{f}$ is the value of the objective function when the updating process has finished).

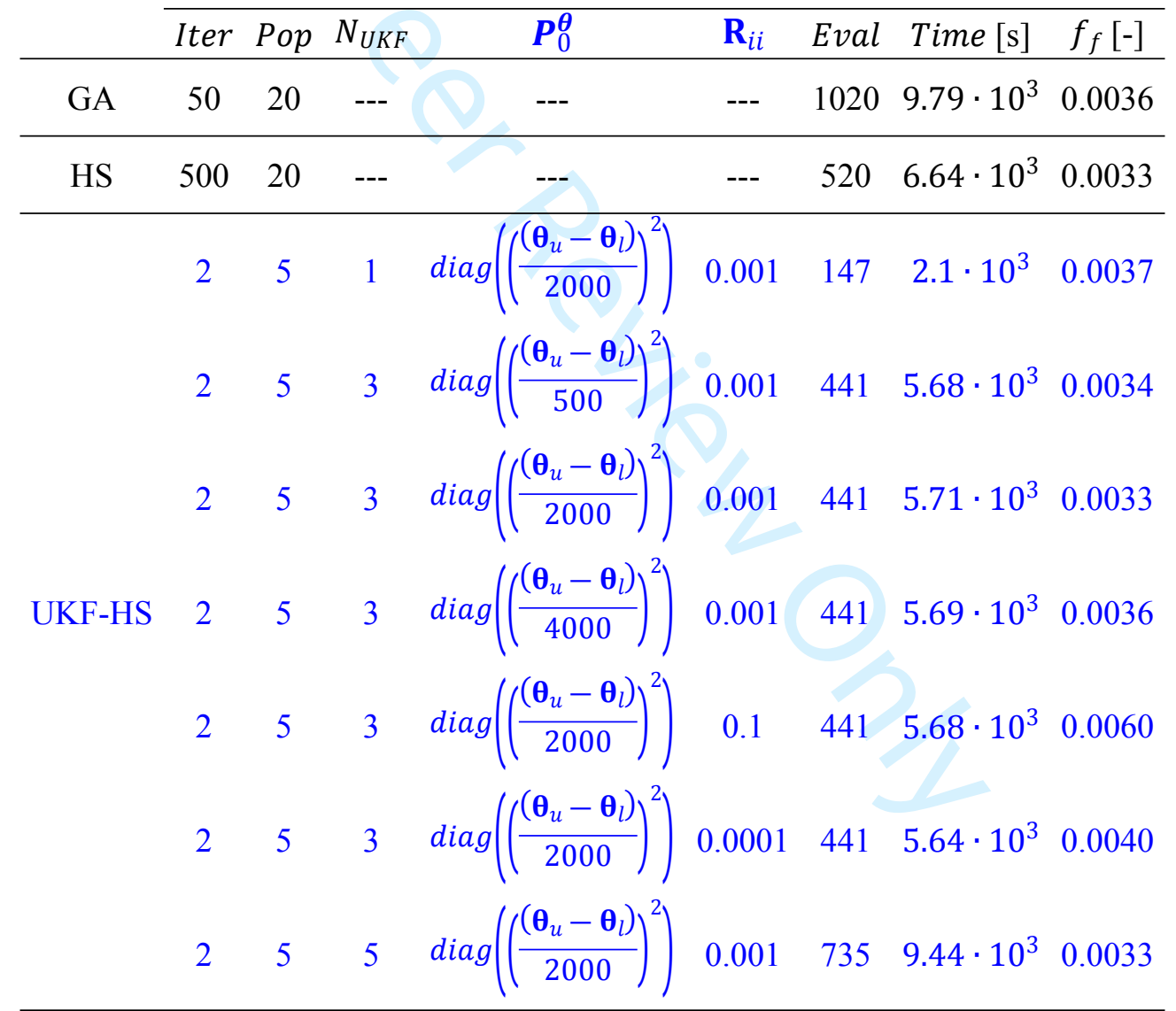


Table 3. Updated value of the physical parameters of model, $\boldsymbol{\theta}$, after the updating process considering the single-objective approach and the three mentioned computational algorithms.

\begin{tabular}{cccc}
\hline $\boldsymbol{\theta}$ & GA & HS & UKF-HS \\
\hline$\theta_{1}$ & $2.29 \cdot 10^{11}$ & $2.28 \cdot 10^{11}$ & $2.29 \cdot 10^{11}$ \\
$\theta_{2}$ & $1.96 \cdot 10^{11}$ & $1.96 \cdot 10^{11}$ & $2.00 \cdot 10^{11}$ \\
$\theta_{3}$ & $2.20 \cdot 10^{11}$ & $2.15 \cdot 10^{11}$ & $2.27 \cdot 10^{11}$ \\
$\theta_{4}$ & $2.04 \cdot 10^{11}$ & $1.91 \cdot 10^{11}$ & $1.97 \cdot 10^{11}$ \\
$\theta_{5}$ & $2.08 \cdot 10^{11}$ & $2.00 \cdot 10^{11}$ & $2.11 \cdot 10^{11}$ \\
$\theta_{6}$ & $2.28 \cdot 10^{11}$ & $2.19 \cdot 10^{11}$ & $2.29 \cdot 10^{11}$ \\
$\theta_{7}$ & $1.04 \cdot 10^{9}$ & $1.28 \cdot 10^{9}$ & $1.29 \cdot 10^{9}$ \\
$\theta_{8}$ & $1.16 \cdot 10^{12}$ & $1.53 \cdot 10^{12}$ & $1.50 \cdot 10^{12}$ \\
$\theta_{9}$ & $7.67 \cdot 10^{7}$ & $7.50 \cdot 10^{7}$ & $7.50 \cdot 10^{7}$ \\
$\theta_{10}$ & $7.66 \cdot 10^{6}$ & $7.72 \cdot 10^{6}$ & $7.73 \cdot 10^{6}$ \\
\hline
\end{tabular}


Table 4. Updated, $f_{u p d, j}$, and experimental, $f_{\text {exp }, j}$, natural frequency, relative difference, $\Delta f_{\text {exp,j }}^{u p, j}$, and the $M A C_{e x p, j}^{u p d . j}$ ratio for each considered vibration mode $j$ after the updating process considering the single-objective approach and the three mentioned computational algorithms.

\begin{tabular}{|c|c|c|c|c|c|c|c|c|c|c|}
\hline \multirow{2}{*}{$\begin{array}{c}\text { Mode } \\
(j)\end{array}$} & \multirow{2}{*}{$\begin{array}{r}f_{\text {exp }, j} \\
{[\mathrm{~Hz}]}\end{array}$} & \multicolumn{3}{|c|}{$\mathbf{G A}$} & \multicolumn{3}{|c|}{ HS } & \multicolumn{3}{|c|}{ UKF-HS } \\
\hline & & $\begin{array}{c}f_{u p d, j} \\
{[\mathrm{~Hz}]}\end{array}$ & $\begin{array}{c}\Delta f_{\text {exp }, j}^{u p d} \\
{[\%]}\end{array}$ & $\begin{array}{c}M A C_{\text {exp }, j}^{u p d . j} \\
{[-]}\end{array}$ & $\begin{array}{r}f_{u p d, j} \\
{[\mathrm{~Hz}]}\end{array}$ & $\begin{array}{c}\Delta f_{e x p, j}^{u p d, j} \\
{[\%]}\end{array}$ & $\begin{array}{c}M A C_{\text {exp.j }}^{u p d . j} \\
{[-]}\end{array}$ & $\begin{array}{c}f_{u p d, j} \\
{[\mathrm{~Hz}]}\end{array}$ & $\begin{array}{c}\Delta f_{\text {exp }, j}^{u p d, j} \\
{[\%]}\end{array}$ & $\begin{array}{c}M A C_{e x p, j}^{u p d . j} \\
{[-]}\end{array}$ \\
\hline 1 & 3.810 & 3.880 & 1.837 & 0.998 & 3.880 & 1.837 & 0.998 & 3.890 & 2.1 & 0.998 \\
\hline 2 & 5.144 & 5.380 & 4.588 & 0.993 & 5.372 & 4.432 & 0.993 & 5.375 & 4.491 & 0.993 \\
\hline 3 & 8.485 & 8.370 & -1.355 & 0.988 & 8.400 & -1.002 & 0.988 & 8.410 & -0.884 & 0.988 \\
\hline 4 & 12.366 & 11.930 & -3.526 & 0.907 & 11.950 & -3.364 & 0.905 & 12.002 & -2.944 & 0.903 \\
\hline 5 & 18.605 & 18.460 & -0.779 & 0.986 & 18.630 & 0.134 & 0.987 & 18.670 & 0.349 & 0.987 \\
\hline 6 & 20.459 & 20,160 & -1.461 & 0.993 & 20.150 & -1.51 & 0.992 & 20.250 & -1.022 & 0.992 \\
\hline 7 & 22.980 & 22,150 & -3.612 & 0.947 & 22.420 & -2.437 & 0.950 & 22.450 & -2.306 & 0.950 \\
\hline
\end{tabular}


Table 5. Performance of the three considered algorithms to solve the updating problem under the multi-objective approach (where Iter is the number of iterations of the main algorithm; Pop is the population size; New Pop is the new population size; $N_{U K F}$ is the number of iterations of the UKF algorithm; $\boldsymbol{P}_{0}^{\boldsymbol{\theta}}$ is the initial estimation error covariance; $\mathbf{R}_{i i}$ is the measurement noise covariance matrix; Eval is the number of evaluations of the objective function; Time is the time [s] required until a convergence criterion is met; Dist is the distance between the selected point and the origin; and Sum is the sum of the two terms of the objective function).

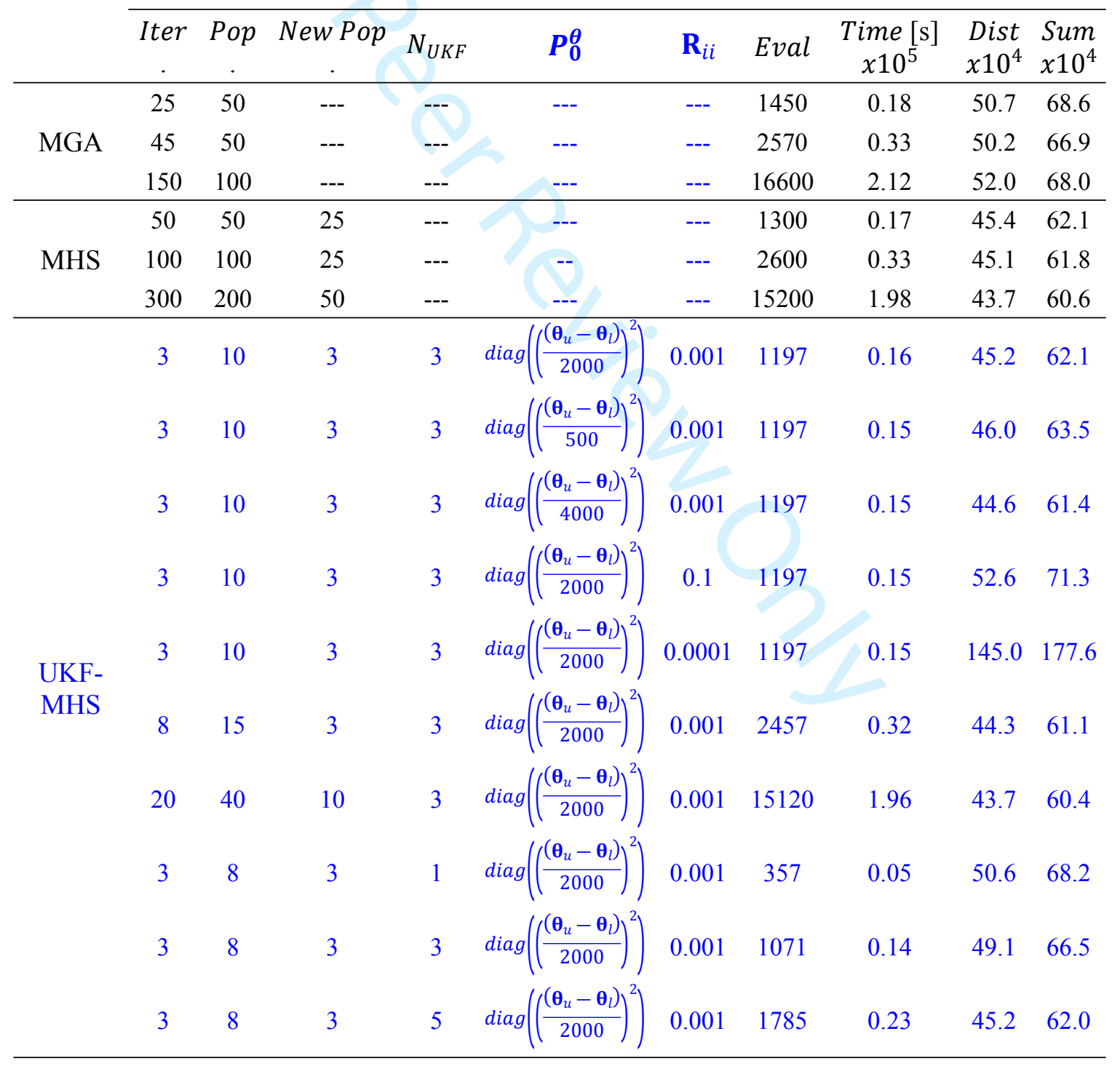


Table 6. Updated value of the physical parameters of model, $\boldsymbol{\theta}$, after the updating process considering the multi-objective approach and the three mentioned computational algorithms.

\begin{tabular}{cccc}
\hline $\boldsymbol{\theta}$ & MGA & MHS & UKF-MHS \\
\hline$\theta_{1}$ & $2.29 \cdot 10^{11}$ & $2.27 \cdot 10^{11}$ & $2.28 \cdot 10^{11}$ \\
$\theta_{2}$ & $2.15 \cdot 10^{11}$ & $2.29 \cdot 10^{11}$ & $2.26 \cdot 10^{11}$ \\
$\theta_{3}$ & $2.06 \cdot 10^{11}$ & $2.22 \cdot 10^{11}$ & $2.26 \cdot 10^{11}$ \\
$\theta_{4}$ & $2.14 \cdot 10^{11}$ & $2.22 \cdot 10^{11}$ & $2.28 \cdot 10^{11}$ \\
$\theta_{5}$ & $2.10 \cdot 10^{11}$ & $1.92 \cdot 10^{11}$ & $2.15 \cdot 10^{11}$ \\
$\theta_{6}$ & $2.02 \cdot 10^{11}$ & $2.26 \cdot 10^{11}$ & $2.17 \cdot 10^{11}$ \\
$\theta_{7}$ & $8.60 \cdot 10^{8}$ & $1.30 \cdot 10^{9}$ & $1.17 \cdot 10^{9}$ \\
$\theta_{8}$ & $7.48 \cdot 10^{11}$ & $1.57 \cdot 10^{12}$ & $1.31 \cdot 10^{12}$ \\
$\theta_{9}$ & $6.43 \cdot 10^{7}$ & $7.50 \cdot 10^{7}$ & $7.50 \cdot 10^{7}$ \\
$\theta_{10}$ & $7.70 \cdot 10^{6}$ & $7.61 \cdot 10^{6}$ & $7.65 \cdot 10^{6}$ \\
\hline
\end{tabular}


Table 7. Updated, $f_{u p d, j}$, and experimental, $f_{\text {exp }, j}$, natural frequency, relative difference, $\Delta f_{\text {exp,j }}^{u p d, j}$, and the $M A C_{\text {exp,j }}^{u p d}$ ratio for each considered vibration mode $j$ after the updating process considering the multi-objective approach and the three mentioned computational algorithms.

\begin{tabular}{|c|c|c|c|c|c|c|c|c|c|c|}
\hline \multirow{2}{*}{$\begin{array}{l}\text { Mode } \\
(j)\end{array}$} & \multirow{2}{*}{$\begin{array}{l}f_{\text {exp, }} \\
{[\mathrm{Hz}]}\end{array}$} & \multicolumn{3}{|c|}{ MGA } & \multicolumn{3}{|c|}{ MHS } & \multicolumn{3}{|c|}{ UKF-MHS } \\
\hline & & $\begin{array}{r}f_{u p d, j} \\
{[\mathrm{~Hz}]}\end{array}$ & $\begin{array}{c}\Delta f_{e x p, j}^{u p d, j} \\
{[\%]}\end{array}$ & $\begin{array}{c}M A C_{e x p, j}^{u p d . j} \\
{[-]}\end{array}$ & $\begin{array}{l}f_{u p d, j} \\
{[\mathrm{~Hz}]}\end{array}$ & $\begin{array}{c}\Delta f_{e x p, j}^{u p d, j} \\
{[\%]}\end{array}$ & $\begin{array}{c}M A C_{e x p, j}^{u p d . j} \\
{[-]}\end{array}$ & $\begin{array}{r}f_{u p d, j} \\
{[\mathrm{~Hz}]}\end{array}$ & $\begin{array}{c}\Delta f_{e x p, j}^{u p d, j} \\
{[\%]}\end{array}$ & $\begin{array}{c}M A C_{e x p, J}^{u p d .} . \\
{[-]}\end{array}$ \\
\hline 1 & 3.810 & 3.900 & 2.362 & 0.998 & 3.871 & 1.601 & 0.998 & 3.872 & 1.627 & 0.999 \\
\hline 2 & 5.144 & 5.283 & 2.702 & 0.994 & 5.270 & 2.449 & 0.994 & 5.252 & 2.100 & 0.994 \\
\hline 3 & 8.485 & 8.373 & -1.320 & 0.988 & 8.360 & -1.473 & 0.988 & 8.361 & -1.461 & 0.989 \\
\hline 4 & 12.366 & 12.040 & -2.636 & 0.902 & 11.933 & -3.502 & 0.902 & 11.862 & -4.076 & 0.901 \\
\hline 5 & 18.605 & 18.360 & -1.317 & 0.985 & 18.630 & 0.134 & 0.987 & 18.479 & -0.677 & 0.987 \\
\hline 6 & 20.459 & 20.471 & 0.059 & 0.993 & 20.100 & -1.755 & 0.993 & 20.003 & -2.229 & 0.993 \\
\hline 7 & 22.980 & 21.880 & -4.787 & 0.951 & 22.420 & -2.437 & 0.951 & 22.234 & -3.246 & 0.948 \\
\hline
\end{tabular}

